\title{
Line Geometry and Camera Autocalibration
}

\author{
José I. Ronda • Antonio Valdés - Guillermo Gallego
}

Cover Date: Oct. 2008

\begin{abstract}
We provide a completely new rigorous matrix formulation of the absolute quadratic complex (AQC), given by the set of lines intersecting the absolute conic. The new results include closed-form expressions for the camera intrinsic parameters in terms of the $\mathrm{AQC}$, an algorithm to obtain the dual absolute quadric from the AQC using straightforward matrix operations, and an equally direct computation of a Euclidean-upgrading homography from the AQC. We also completely characterize the $6 \times 6$ matrices acting on lines which are induced by a spatial homography.

Several algorithmic possibilities arising from the AQC are systematically explored and analyzed in terms of efficiency and computational cost. Experiments include $3 \mathrm{D}$ reconstruction from real images.
\end{abstract}

Keywords Camera autocalibration · Varying parameters $\cdot$ Absolute quadratic complex

\section{Introduction}

The problem of obtaining a $3 \mathrm{D}$ reconstruction from a set of images is a central issue in modern computer vision $[10,11,16]$. An important practical situation is that in which no a priori knowledge of the scene and camera positions is available and the data about the camera internal parameters is reduced to a minimum. This is called the autocalibration problem. If nothing is known

J.I. Ronda $(\varangle)$ · G. Gallego

Grupo de Tratamiento de Imágenes, Universidad Politécnica de Madrid, 28040 Madrid, Spain

E-mail: jir@gti.ssr.upm.es, ggb@gti.ssr.upm.es

A. Valdés

Dep. de Geometría y Topología, Universidad Complutense de Madrid, 28040 Madrid, Spain

E-mail: Antonio_Valdes@mat.ucm.es about the internal parameters of the cameras then only a projective reconstruction is possible, i.e., one that differs from the actual scene in a $3 \mathrm{D}$ homography $[7,12]$.

Two kinds of restrictions in the camera internal parameters leading to Euclidean reconstructions have been mainly considered in the literature. The first one is that of cameras with constant internal parameters [17]. To solve the autocalibration problem in this case, the main geometrical object used was the absolute conic [25], which is an imaginary conic on the plane at infinity encoding the Euclidean structure of 3D space. To obtain the image of the absolute conic (IAC) Kruppa equations were employed.

An alternative to Kruppa equations is the unimodular constraint [18], expressing the fact that the interimage homographies induced by the plane at infinity are conjugated to rotation matrices. This allows for the obtainment of the plane at infinity as an intermediate step called affine calibration. The resulting approach is termed stratified calibration.

Another geometrical object equivalent to the absolute conic is the dual absolute quadric (DAQ) [29], given by the set of planes tangent to the absolute conic. With respect to the absolute conic the DAQ has the advantage of being given by a single equation, being particularly useful to profit from the knowledge of orthogonal plane pairs.

The second kind of restriction is used in the case of cameras with variable parameters, consisting in restrictions of some camera parameters as the pixel shape. For example, in [1] the inter-image homography is considered for the autocalibration of purely rotating cameras with varying parameters. This homography relates the images taken with two cameras with the same projection center and thus the associated IAC's. Linear and 
non-linear algorithms are provided for the case of cameras with rectangular or square pixels.

Another instance in which the 3D reconstruction is obtained in a particularly simple way is that in which the principal point positions are known along with the fact that the camera has square pixels. In fact, using the DAQ, this results in a set of linear equations $[19$, 26]. Another alternative for this problem is proposed in [27], consisting in an iterative algorithm to improve an initial guess on the principal point position.

The characterization given in $[8$, p. 53] and [13] of square pixel cameras has been used in [5] and [14] to calibrate cameras with varying parameters through the minimization of a cost function in terms of the projection matrices. The initialization of the algorithms were based either on a priori approximate knowledge of the internal parameters or on the assumption of constant internal parameters so that Kruppa equations can be used. In [19] the same problem is adressed through the optimization of a cost function depending on the DAQ and the intrinsic camera parameters. Critical motions for autocalibration of cameras with fixed aspect ratio and skew but with other parameters varying are studied in [15].

All the aforementioned algorithms for autocalibration of cameras with varying parameters under general motion share the limitation of needing an accurate initialization with an approximate solution.

The above-mentioned characterization of projection matrices for cameras with rectangular pixels was also the starting point in [20] to obtain the matrix establishing the orthogonality of lines in Plücker coordinates. This matrix was introduced as a geometric object on its own in [21], calling it the Absolute Quadratic Complex (AQC).

The AQC is given by the set of lines that intersect the absolute conic $[20,21,30-32]$, sharing with the DAQ the advantage over the absolute conic of being given by a single equation. An advance of some results presented in this paper appeared in conference paper [24]. The AQC is particularly well-suited for autocalibration when pairs of orthogonal lines are known and also in the case of square-pixel cameras, because then each camera provides two lines intersecting the absolute conic, leading to linear equations in the AQC parameters. This solves, when enough cameras are available, the initialization problem of previous techniques.

Handling the set of lines in 3D space is of interest not only in relation with the AQC but also in other computer vision problems, such as $3 \mathrm{D}$ reconstruction based on line correspondences [2]. In this work Plücker coordinates were employed, but other possible techniques to deal with lines in 3D space are the double-algebra theory as described, e.g., in [6,9], Clifford Algebras [3] or exterior algebra, which is used, e.g., in [32]. The advantage of the Plücker matrix approach of this paper is that it is very close to the implementation of the algorithms.

One of our aims is to provide a completely new rigorous matrix formulation of the AQC. An effort has been made to make the paper self-contained. With this purpose we include an introduction to Plücker theory, covering several aspects which are not easily available in the literature but are necessary for the AQC theory.

New properties of the AQC are also presented and exploited to obtain novel autocalibration algorithms. The new results include closed-form expressions for the camera intrinsic parameters in terms of the AQC (Sect. 3.8 ), an algorithm to obtain the DAQ from the AQC using straightforward matrix operations (Sect. 3.9), and an equally direct computation of a Euclidean-upgrading homography (Sect. 3.10) from the AQC, formalizing a technique proposed in [20]. We also completely characterize the $6 \times 6$ matrices acting on lines which are induced by a spatial homography (equations (27)), completing a previous result given in [2]. A mathematical proof of the fact that the operation attaching to each spatial homography its line homography is invariant under transposition is given as well.

In the algorithmic part of this work, several possibilities arising from the AQC are systematically explored and analyzed in terms of efficiency and computational cost. The potential of the AQC to provide accurate initializations is exploited. Experiments include testing with three different sets of real data and comparison with other algorithms.

The paper is organized as follows. Section 2 introduces the required tools in order to deal with geometry of lines in space. Then Sect. 3 introduces the AQC in a new way that makes explicit its relationship with the DAQ. Section 4 addresses the relevance of the AQC in the case of cameras with known pixel shape. The new autocalibration techniques that arise from this work are presented and tested with synthetic and real data in Sect. 5. Conclusions are provided in Sect. 6. Proofs of some of the mathematical results of the paper are postponed to the Appendix.

\section{Line representation}

Plücker coordinates $[22,25]$ are a very convenient mathematical representation of lines in 3D space. The core of Plücker theory is the existence of two natural one-toone correspondences between lines of space and the set of rank-two $4 \times 4$ antisymmetric matrices. In this section we summarize the notation and results of Plücker 
theory that will be relevant in the rest of the paper. Our presentation covers many aspects which are not available in the literature and are necessary for the AQC theory. Proofs are postponed to the Appendix.

\subsection{Plücker matrices}

Given two vectors $\mathbf{u}=\left(u_{1}, u_{2}, u_{3}, u_{4}\right)^{\top}, \mathbf{v}=\left(v_{1}, v_{2}, v_{3}\right.$, $\left.v_{4}\right)^{\top} \in \mathbb{C}^{4}$, we define the antisymmetric matrix

$\mathrm{M}(\mathbf{u}, \mathbf{v})=\mathbf{u v}^{\top}-\mathbf{v} \mathbf{u}^{\top}=\left(\begin{array}{cccc}0 & m_{12} & m_{13} & m_{14} \\ -m_{12} & 0 & m_{23} & m_{24} \\ -m_{13} & -m_{23} & 0 & m_{34} \\ -m_{14} & -m_{24} & -m_{34} & 0\end{array}\right)$,

$m_{i j}=u_{i} v_{j}-u_{j} v_{i}$.

Note that $\mathrm{M}(\mathbf{u}, \mathbf{v})=0$ if and only if $\mathbf{u}$ and $\mathbf{v}$ are dependent, and otherwise the rank of $\mathrm{M}(\mathbf{u}, \mathbf{v})$ is two. The L-matrix of a line $l$ is given by

$\mathrm{L}=\mathrm{M}(\mathbf{p}, \mathbf{q})$.

where $\mathbf{p}$ and $\mathbf{q}$ are any two points of $l$. Changing the points leads to a proportional matrix, so that L-matrices are defined up to a non-zero scale.

\subsubsection{Basic relations}

The planes $\boldsymbol{\alpha}$ containing the line are those satisfying

$\mathrm{L} \boldsymbol{\alpha}=0$.

Therefore each line is determined by its L-matrix.

Since L-matrices are singular, they satisfy

$\operatorname{det} \mathrm{L}=\left(m_{12} m_{34}+m_{13} m_{42}+m_{14} m_{23}\right)^{2}=0$.

Conversely, since singular antisymmetric matrices are defined up to a constant factor by their kernel (remarks A1 and A2 in Sect. A.1), any singular $4 \times 4$ nonzero antisymmetric matrix turns out to be the L-matrix of some line.

The intersection of the line $l$ and the plane $\boldsymbol{\alpha}$ is given by

$\mathrm{X}=\mathrm{L} \boldsymbol{\alpha}$.

Along with the L-matrix, we can also assign a line its $\mathrm{L}^{*}$-matrix, given by

$\mathrm{L}^{*}=\mathrm{M}(\boldsymbol{\alpha}, \boldsymbol{\beta})$,

where $\boldsymbol{\alpha}$ and $\boldsymbol{\beta}$ are two planes defining the line. The properties of this matrix are dual versions of those of L-matrices. In particular, $\mathrm{L}^{*}$-matrices characterize the set of points of the line by the relation $L^{*} \mathbf{X}=0$, and the plane $\boldsymbol{\gamma}$ defined by the line and an external point $\mathbf{X}$ is obtained as $\gamma=\mathrm{L}^{*} \mathbf{X}$. The L-matrix and the $\mathrm{L}^{*}$-matrix of a line will be called its Plücker matrices. Next, we will show the relationship between them.

Given two vectors $\mathbf{u}, \mathbf{v} \in \mathbb{C}^{4}$, define the matrix $\mathbf{M}^{*}(\mathbf{u}, \mathbf{v})$ as the only one satisfying

$\mathbf{x}^{\top} \mathrm{M}^{*}(\mathbf{u}, \mathbf{v}) \mathbf{y}=\operatorname{det}(\mathbf{x}, \mathbf{u}, \mathbf{v}, \mathbf{y})$

for any vectors $\mathbf{x}, \mathbf{y}$. Its explicit expression is

$\mathbf{M}^{*}(\mathbf{u}, \mathbf{v})=\left(\begin{array}{cccc}0 & m_{34} & m_{42} & m_{23} \\ -m_{34} & 0 & m_{14} & m_{31} \\ -m_{42} & -m_{14} & 0 & m_{12} \\ -m_{23} & -m_{31} & -m_{12} & 0\end{array}\right)$

with $m_{i j}$ defined as in (1). Therefore $\mathbf{M}^{*}(\mathbf{u}, \mathbf{v})$ can be obtained from $\mathrm{M}(\mathbf{u}, \mathbf{v})$ by certain transpositions of coefficients, so that $\mathrm{M}^{* *}=\mathrm{M}$. Given the points $\mathbf{p}$, $\mathbf{q}$, the kernel of the matrix $\mathrm{M}^{*}(\mathbf{p}, \mathbf{q})$ is, as a consequence of (7), the set of points of the line through them. Therefore, $\mathrm{M}^{*}(\mathbf{p}, \mathbf{q})$ coincides with the $\mathrm{L}^{*}$-matrix of the line. Dually, if $\boldsymbol{\alpha}$ and $\boldsymbol{\beta}$ are two planes, $\mathrm{M}^{*}(\boldsymbol{\alpha}, \boldsymbol{\beta})$ is a L-matrix of the line defined by them. Hence, $\mathrm{M}^{*}(\boldsymbol{\alpha}, \boldsymbol{\beta}) \sim \mathrm{M}(\mathbf{p}, \mathbf{q})$.

\subsubsection{Incidence of lines.}

Incidence between lines is easily established in terms of Plücker matrices (see Sect. A.2). Two lines $l_{1}$ and $l_{2}$, with matrices $L_{1}$ and $L_{2}^{*}$ respectively, intersect if and only if

$\operatorname{trace}\left(\mathrm{L}_{1} \mathrm{~L}_{2}^{*}\right)=0$.

If the two lines intersect, any non-zero column of the product $\mathrm{L}_{1} \mathrm{~L}_{2}^{*}$ represents the intersection point. Analogously, any non-zero row of the product $\mathrm{L}_{1} \mathrm{~L}_{2}^{*}$ represents the common plane. Finally, since a line intersects itself, for any L-matrix,

$\operatorname{trace}\left(\mathrm{LL}^{*}\right)=0$,

but this condition is just (4).

Three lines are concurrent when the intersection of two of them lies on the third one or, equivalently, when $\mathrm{L}_{1}^{*} \mathrm{~L}_{2} \mathrm{~L}_{3}^{*}=0$. Dually, the coplanarity of three lines is characterized by equation $\mathrm{L}_{1} \mathrm{~L}_{2}^{*} \mathrm{~L}_{3}=0$. Table 1 summarizes previous formulas.

\subsubsection{Changes of coordinates}

Consider the change of coordinates (or the linear mapping) in $\mathbb{P}^{3}$ given by $\mathbf{p}^{\prime}=$ Hp. If the line $l$ is defined by points $\mathbf{p}, \mathbf{q}$, its associated L-matrix will be given in the new coordinate system by

$\mathrm{M}\left(\mathbf{p}^{\prime}, \mathbf{q}^{\prime}\right)=\mathrm{HM}(\mathbf{p}, \mathbf{q}) \mathrm{H}^{\top}$. 
Table 1 Operations with points, planes and lines using Plücker matrices.

\begin{tabular}{llll}
\hline Line defined by & $\mathrm{L}=\mathrm{M}(\mathbf{p}, \mathbf{q})$ & Line defined by & $\mathrm{L}=\mathrm{M}^{*}(\boldsymbol{\alpha}, \boldsymbol{\beta})$ \\
two points & & two planes & \\
Point on the line & $\mathrm{L}^{*} \mathbf{X}=\mathbf{0}$ & Plane containing the line & $\mathrm{L} \boldsymbol{\pi}=\mathbf{0}$ \\
Plane defined by point and line & $\boldsymbol{\pi}=\mathrm{L}^{*} \mathbf{X}$ & Point defined by plane and line & $\mathbf{X}=\mathrm{L} \boldsymbol{\pi}$ \\
Plane defined by & $\boldsymbol{\pi}=\operatorname{row}\left(\mathrm{L}_{1} \mathrm{~L}_{2}^{*}\right)$ & Point defined by & $\mathbf{X}=\operatorname{col}\left(\mathrm{L}_{1} \mathrm{~L}_{2}^{*}\right)$ \\
two coplanar lines & $=\operatorname{col}\left(\mathrm{L}_{1}^{*} \mathrm{~L}_{2}\right)$ & two coplanar lines & $=\operatorname{row}\left(\mathrm{L}_{1}^{*} \mathrm{~L}_{2}\right)$ \\
Plane through three points & $\boldsymbol{\pi}=\mathrm{M}^{*}(\mathbf{p}, \mathbf{q}) \mathbf{r}$ & Point through three planes & $\mathbf{X}=\mathrm{M}^{*}(\boldsymbol{\alpha}, \boldsymbol{\beta}) \boldsymbol{\gamma}$ \\
Three lines are coplanar & $\mathrm{L}_{1} \mathrm{~L}_{2}^{*} \mathrm{~L}_{3}=0$ & Three lines are concurrent & $\mathrm{L}_{1}^{*} \mathrm{~L}_{2} \mathrm{~L}_{3}^{*}=0$ \\
\hline
\end{tabular}

Analogously, the corresponding change of coordinates for planes $\boldsymbol{\alpha}^{\prime}=\mathrm{H}^{-\top} \boldsymbol{\alpha}$ induces the transformation formula for $\mathrm{L}^{*}$-matrices $\mathrm{M}\left(\boldsymbol{\alpha}^{\prime}, \boldsymbol{\beta}^{\prime}\right)=\mathrm{H}^{-\top} \mathrm{M}(\boldsymbol{\alpha}, \boldsymbol{\beta}) \mathrm{H}^{-1}$. But since $\mathrm{M}^{*}(\mathbf{p}, \mathbf{q}) \sim \mathrm{M}(\boldsymbol{\alpha}, \boldsymbol{\beta})$ and $\mathrm{M}^{*}\left(\mathbf{p}^{\prime}, \mathbf{q}^{\prime}\right) \sim \mathrm{M}\left(\boldsymbol{\alpha}^{\prime}, \boldsymbol{\beta}^{\prime}\right)$, we obtain

$$
\begin{aligned}
\mathrm{M}^{*}(\mathrm{H} \mathbf{p}, \mathrm{Hq}) & =\rho_{1} \mathrm{M}\left(\mathrm{H}^{-\top} \boldsymbol{\alpha}, \mathrm{H}^{-\top} \boldsymbol{\beta}\right)=\rho_{1} \mathrm{H}^{-\top} \mathrm{M}(\boldsymbol{\alpha}, \boldsymbol{\beta}) \mathrm{H}^{-1} \\
& =\rho_{1} \rho_{2} \mathrm{H}^{-\top} \mathrm{M}^{*}(\mathbf{p}, \mathbf{q}) \mathrm{H}^{-1}
\end{aligned}
$$

for some scalars $\rho_{1}, \rho_{2}$. The proportionality constant $\rho=\rho_{1} \rho_{2}$ can be obtained as follows. From (7) we have

$$
\begin{aligned}
(\mathrm{H} \mathbf{x})^{\top} \mathrm{M}^{*}(\mathrm{H} \mathbf{p}, \mathrm{Hq})(\mathrm{Hy}) & =\operatorname{det}(\mathrm{H} \mathbf{x}, \mathrm{H} \mathbf{p}, \mathrm{Hq}, \mathrm{Hy}) \\
& =\operatorname{det}(\mathrm{H}) \operatorname{det}(\mathbf{x}, \mathbf{p}, \mathbf{q}, \mathbf{y}) .
\end{aligned}
$$

And, from (12), the left-hand side of this last equation is

$$
\begin{aligned}
(\mathrm{H} \mathbf{x})^{\top}\left(\rho \mathrm{H}^{-\top} \mathrm{M}^{*}(\mathbf{p}, \mathbf{q}) \mathrm{H}^{-1}\right)(\mathrm{Hy}) & =\rho \mathbf{x}^{\top} \mathrm{M}^{*}(\mathbf{p}, \mathbf{q}) \mathbf{y} \\
& =\rho \operatorname{det}(\mathbf{x}, \mathbf{p}, \mathbf{q}, \mathbf{y}),
\end{aligned}
$$

so that $\rho=\operatorname{det}(\mathrm{H})$, i.e.,

$\mathrm{M}^{*}(\mathrm{H} \mathbf{p}, \mathrm{Hq})=\operatorname{det}(\mathrm{H}) \mathrm{H}^{-\top} \mathrm{M}^{*}(\mathbf{p}, \mathbf{q}) \mathrm{H}^{-1}$.

\subsection{Plücker coordinates}

A convenient choice of basis of the set of $4 \times 4$ antisymmetric matrices is

$$
\begin{aligned}
\mathrm{B}= & \left\{\mathrm{M}\left(\mathbf{e}_{3}, \mathbf{e}_{4}\right), \mathrm{M}\left(\mathbf{e}_{1}, \mathbf{e}_{4}\right), \mathrm{M}\left(\mathbf{e}_{2}, \mathbf{e}_{4}\right), \mathrm{M}\left(\mathbf{e}_{3}, \mathbf{e}_{1}\right),\right. \\
& \left.\mathrm{M}\left(\mathbf{e}_{2}, \mathbf{e}_{3}\right), \mathrm{M}\left(\mathbf{e}_{1}, \mathbf{e}_{2}\right)\right\} \\
= & \left\{\mathrm{M}^{*}\left(\mathbf{e}_{1}, \mathbf{e}_{2}\right), \mathrm{M}^{*}\left(\mathbf{e}_{2}, \mathbf{e}_{3}\right), \mathrm{M}^{*}\left(\mathbf{e}_{3}, \mathbf{e}_{1}\right), \mathrm{M}^{*}\left(\mathbf{e}_{2}, \mathbf{e}_{4}\right),\right. \\
& \left.\mathrm{M}^{*}\left(\mathbf{e}_{1}, \mathbf{e}_{4}\right), \mathrm{M}^{*}\left(\mathbf{e}_{3}, \mathbf{e}_{4}\right)\right\},
\end{aligned}
$$

so that an antisymmetric matrix $\mathrm{A}=\left(a_{i j}\right)$ will have coordinates with respect to $B$

$\boldsymbol{\ell}_{\mathrm{A}}=\left(a_{34}, a_{14}, a_{24}, a_{31}, a_{23}, a_{12}\right)^{\top}$.

Note that given antisymmetric matrices A, B, we have

$\frac{1}{2} \operatorname{trace}\left(\mathrm{A}^{\top} \mathrm{B}\right)=\boldsymbol{\ell}_{\mathrm{A}}^{\top} \boldsymbol{\ell}_{\mathrm{B}}$.
The mapping $\mathrm{M} \mapsto \mathrm{M}^{*}$ given in (8) corresponds to

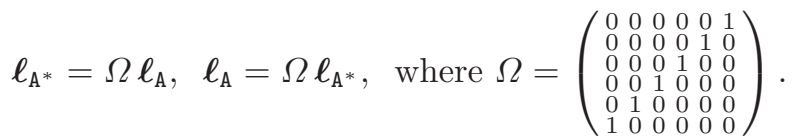

This accounts for the ordering of the elements of the basis $B$.

We define the Plücker coordinates of a line as the coordinates of its L-matrix with respect to $\mathrm{B}$, so if a line $l$ is given by points $\mathbf{p}, \mathbf{q}$ or by planes $\boldsymbol{\alpha}, \boldsymbol{\beta}$, its Plücker coordinates $\ell$ are

$\ell \sim \ell_{\mathrm{M}(\mathbf{p}, \mathbf{q})} \sim \ell_{\mathrm{M}^{*}(\boldsymbol{\alpha}, \boldsymbol{\beta})}$.

Relations (16) and (17) allow for an easy translation of previous formulas involving Plücker matrices to the language of Plücker coordinates. In particular, according to (10), a non-zero vector $\ell \in \mathbb{C}^{6}$ will correspond to the Plücker coordinates of some line if and only if

$\ell^{\top} \Omega \ell=0$.

The quadric with matrix $\Omega$ is known as the Klein quadric.

The incidence relation (9) in terms of Plücker coordinates is given by

$\frac{1}{2} \operatorname{trace}\left(\mathrm{L}_{1}^{\top} \mathrm{L}_{2}^{*}\right)=\boldsymbol{\ell}_{\mathrm{L}_{1}}^{\top} \Omega \boldsymbol{\ell}_{\mathrm{L}_{2}}=0$,

due to (16) and (17). Therefore, two lines intersect if and only if their Plücker coordinates are conjugate with respect to the Klein quadric.

Given vectors $\mathbf{u}, \mathbf{v}$ of $\mathbb{C}^{4}$, we define

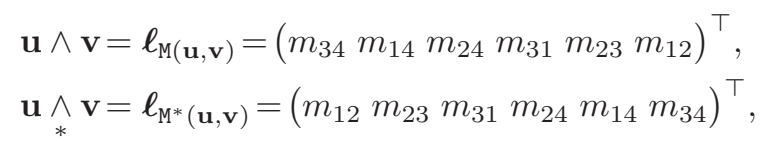

where $m_{i j}=u_{i} v_{j}-u_{j} v_{i}$. It is immediate that these operations are antisymmetric and bilinear. Thus, if $\boldsymbol{\alpha}, \boldsymbol{\beta}$ represent planes defining the line $l$ through the points $\mathbf{p}, \mathbf{q}$, then $\mathbf{p} \wedge \mathbf{q} \sim \boldsymbol{\alpha} \wedge_{*} \boldsymbol{\beta}$ are the Plücker coordinates of $l$. 
From (17) and (21) it follows that

$\Omega(\mathbf{u} \wedge \mathbf{v})=\mathbf{u} \wedge \mathbf{v}, \quad \Omega\left(\mathbf{u} \wedge_{*} \mathbf{v}\right)=\mathbf{u} \wedge \mathbf{v}$,

so that

$\Omega^{2}=\mathrm{I}$.

\subsubsection{Changes of coordinates}

Changes of coordinates of $\mathbb{P}^{3}$ affect Plücker coordinates according to a relationship deriving from (11). The change of coordinates of $\mathbb{P}^{3}$ given by $\mathbf{p}^{\prime}=\mathrm{H} \mathbf{p}$, and therefore $\boldsymbol{\alpha}^{\prime}=\mathrm{H}^{-\top} \boldsymbol{\alpha}$, induces the change of Plücker coordinates

$$
\begin{aligned}
\boldsymbol{\ell}_{\mathrm{M}\left(\mathbf{p}^{\prime}, \mathbf{q}^{\prime}\right)} & =\tilde{\mathrm{H}} \boldsymbol{\ell}_{\mathrm{M}(\mathbf{p}, \mathbf{q})}, \\
\boldsymbol{\ell}_{\mathrm{M}^{*}\left(\boldsymbol{\alpha}^{\prime}, \boldsymbol{\beta}^{\prime}\right)} & =\operatorname{det}\left(\mathrm{H}^{-1}\right) \tilde{\mathrm{H}} \boldsymbol{\ell}_{\mathrm{M}^{*}(\boldsymbol{\alpha}, \boldsymbol{\beta})},
\end{aligned}
$$

where, being $\mathbf{h}_{i}$ the columns of $\mathrm{H}$ for $i=1, \ldots, 4$,

$$
\begin{aligned}
\tilde{\mathrm{H}}=\left(\mathbf{h}_{3} \wedge \mathbf{h}_{4} \mathbf{h}_{1} \wedge \mathbf{h}_{4} \quad \mathbf{h}_{2} \wedge \mathbf{h}_{4} \mathbf{h}_{3} \wedge \mathbf{h}_{1}\right. \\
\left.\mathbf{h}_{2} \wedge \mathbf{h}_{3} \mathbf{h}_{1} \wedge \mathbf{h}_{2}\right)
\end{aligned}
$$

(these formulas and the following ones are proved in Sect. A.3). The matrices of this form have the property

$\tilde{\mathrm{H}}^{\top} \Omega \tilde{\mathrm{H}}=\operatorname{det}(\mathrm{H}) \Omega$.

In fact, a necessary and sufficient condition for a $6 \times 6$ matrix $\mathrm{A}=\left(\mathbf{a}_{1}, \ldots, \mathbf{a}_{6}\right)$ to be of the form $\mathrm{A}=\tilde{\mathrm{H}}$ is that

$$
\begin{aligned}
\mathrm{A}^{\top} \Omega \mathrm{A} & \sim \Omega \\
\mathrm{L}_{1}^{*} \mathrm{~L}_{2} \mathrm{~L}_{3}^{*} & =0,
\end{aligned}
$$

where the $\mathrm{L}_{i}$ are the Plücker matrices defined by the condition $\ell_{\mathrm{L}_{i}}=\mathbf{a}_{i}$ (see Sect. A.3, cf. [2]). Formula (26) holds true also for singular matrices.

It is known that duality switches points and planes in 3D-space. The self-duality of lines is nicely encoded in the next formula, which is also proved in the Appendix (cf. [2]):

$\tilde{\mathrm{H}}^{\top}=\widetilde{\mathrm{H}^{\top}}$.

Another useful formula, that is immediate from the definition of $\tilde{\mathrm{H}}$, is

$\tilde{\mathrm{H}}^{-1}=\widetilde{\mathrm{H}^{-1}}$.

\subsubsection{Plücker coordinates and projections}

Plücker coordinates allow us to express in a practical way two projection relationships involving lines (see Sect. A.5). The projection of a spatial line $\boldsymbol{\ell}$ through a camera given by a projection matrix $\mathrm{P}=\left(\boldsymbol{\pi}_{1}, \boldsymbol{\pi}_{2}, \boldsymbol{\pi}_{3}\right)^{\top}$ is

$\mathbf{r}=\mathcal{P} \Omega \boldsymbol{\ell}$ where $\mathcal{P}=\left(\boldsymbol{\pi}_{2}{\underset{*}{*}}_{\boldsymbol{\pi}_{3}} \boldsymbol{\pi}_{3}{\underset{*}{\wedge}}_{\boldsymbol{\pi}_{1}} \boldsymbol{\pi}_{1}{\underset{*}{*}}_{\boldsymbol{\pi}_{2}}\right)^{\top}$.

The back-projected line of a point $\mathbf{x}$ in the image plane has Plücker coordinates

$\ell=\mathcal{P}^{\top} \mathbf{x}$

cf. [9, p. 194], [21].

\section{The absolute quadratic complex}

\subsection{Introducing the absolute quadratic complex}

Recall that the dual absolute quadric (DAQ) $Q_{\infty}^{*}$ is a positive-semidefinite rank-three $4 \times 4$ symmetric matrix that can be seen as a mapping that assigns to each plane $\boldsymbol{\alpha}$ the point at infinity $\mathbf{X}=\mathbf{Q}_{\infty}^{*} \boldsymbol{\alpha}$, corresponding to its orthogonal vector [29]. Let us consider a line $l$ given by the planes $\boldsymbol{\alpha}$ and $\boldsymbol{\beta}$, and not contained in the plane at infinity, $\boldsymbol{\pi}_{\infty}$. The line $l^{\perp}$ of $\boldsymbol{\pi}_{\infty}$ joining points $\mathbf{Q}_{\infty}^{*} \boldsymbol{\alpha}$ and $\mathrm{Q}_{\infty}^{*} \boldsymbol{\beta}$ is the set of orthogonal directions to $l$. Therefore, the L-matrix of $l^{\perp}$ is

$$
\begin{aligned}
\mathrm{L}^{\perp} & =\mathrm{M}\left(\mathrm{Q}_{\infty}^{*} \boldsymbol{\alpha}, \mathrm{Q}_{\infty}^{*} \boldsymbol{\beta}\right)=\mathrm{Q}_{\infty}^{*}\left(\boldsymbol{\alpha} \boldsymbol{\beta}^{\top}-\boldsymbol{\beta} \boldsymbol{\alpha}^{\top}\right) \mathrm{Q}_{\infty}^{*} \\
& =\mathrm{Q}_{\infty}^{*} \mathrm{~L}^{*} \mathrm{Q}_{\infty}^{*} .
\end{aligned}
$$

where $\mathrm{L}^{*}=\mathrm{M}(\boldsymbol{\alpha}, \boldsymbol{\beta})$ is the $\mathrm{L}^{*}$-matrix of $l$. Note that if $l$ is contained in $\boldsymbol{\pi}_{\infty}$ then we can take $\boldsymbol{\alpha}=\boldsymbol{\pi}_{\infty}$ and since $\mathrm{Q}_{\infty}^{*} \boldsymbol{\pi}_{\infty}=\mathbf{0}$ it results $\mathrm{L}^{\perp}=\mathbf{0}$, which is consistent with the fact that the orthogonal line is not defined for lines at infinity, which in turn define an orthogonal point.

Two coplanar lines not in $\boldsymbol{\pi}_{\infty}, l$ and $l^{\prime}$, are orthogonal if and only if $l^{\perp}$ intersects $l^{\prime}$, i.e., using (9), if

$\operatorname{trace}\left(\mathrm{L}^{* \prime} \mathrm{L}^{\perp}\right)=\operatorname{trace}\left(\mathrm{L}^{* \prime} \mathrm{Q}_{\infty}^{*} \mathrm{~L}^{*} \mathrm{Q}_{\infty}^{*}\right)=0$.

The line $l^{\perp}$ being the polar line with respect to the absolute conic of the point at infinity $\mathbf{p}_{\infty}$ of $l$, we have that the lines not in $\boldsymbol{\pi}_{\infty}$ that intersect the absolute conic are exactly those that intersect their own orthogonal line (see Fig. 1). We will call such lines isotropic lines. Therefore isotropic lines are characterized by the equation

$\operatorname{trace}\left(L^{*} Q_{\infty}^{*} L^{*} Q_{\infty}^{*}\right)=\operatorname{trace}\left[\left(L^{*} Q_{\infty}^{*}\right)^{2}\right]=0$. 


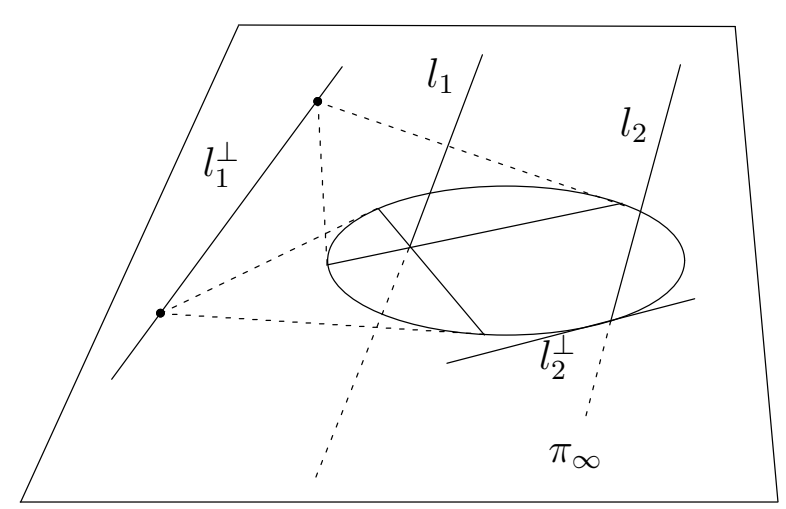

Fig. 1 Incidence of lines with the absolute conic.

This is a quadratic expression in the coordinates of $\mathrm{L}^{*}$ which will be called the absolute quadratic complex (AQC).

The AQC allows to express the Euclidean structure of space in an alternative way to the DAQ. In the same way as the DAQ is given by the tangent planes to the absolute conic, the AQC is given by the set of lines that intersect it.

\subsection{The AQC in terms of Plücker coordinates}

Note that (33) is a bilinear symmetric expression in $\mathrm{L}^{*}$ and $\mathrm{L}^{* \prime}$. Hence, some $6 \times 6$ symmetric matrix $\Sigma$ exists so that

$\frac{1}{2} \operatorname{trace}\left(\left(\mathrm{L}^{* \prime}\right)^{\top} \mathrm{Q}_{\infty}^{*} \mathrm{~L}^{*} \mathrm{Q}_{\infty}^{*}\right)=\boldsymbol{\ell}_{\mathrm{L}^{\prime}}^{\top} \Sigma \boldsymbol{\ell}_{\mathrm{L}}$

Therefore, two coplanar lines $l$ and $l^{\prime}$, are orthogonal if and only if

$\ell^{\prime \top} \Sigma \ell=0$

Likewise, from (34) and (35) $l$ intersects the absolute conic if and only if

$\ell^{\top} \Sigma \ell=0$.

Notice that

$l^{\prime} \perp l \Leftrightarrow$

$\ell^{\prime \top} \Sigma \boldsymbol{\ell}=0 \Leftrightarrow\left(\right.$ since $\left.\Omega^{2}=\mathrm{I}\right)$

$\ell^{\prime \top} \Omega(\Omega \Sigma \ell)=0$.

Last equation is equivalent to $\ell^{\top} \Omega \ell^{\perp}=0$ for any $l^{\prime}$ and therefore $\Omega \Sigma \boldsymbol{\ell}=\ell^{\perp}$. In particular, applying $\Omega \Sigma$ to the canonical basis of $\mathbf{C}^{6}$ (which are indeed Plücker coordinates of lines) we conclude that the columns of $\Omega \Sigma$ are also Plücker coordinates of lines that span the lines contained in $\boldsymbol{\pi}_{\infty}$. Then, the columns of $\Omega \Sigma$ satisfy relation (19) or, equivalently, $\Sigma$ satisfies

$\Sigma \Omega \Sigma=0$.
Besides, we see that $\Sigma$ is a rank-three matrix, since the lines of a plane constitute a linear subspace of $\mathbf{C}^{6}$ of dimension three. Furthermore, the kernel of $\Sigma$ consists of the set of lines contained in the plane at infinity. To see this, observe from (39) that $\Sigma \Omega \Sigma \boldsymbol{\ell}=0$ for any $\boldsymbol{\ell}$. Since $\Omega \Sigma \boldsymbol{\ell}$ can be any line at $\boldsymbol{\pi}_{\infty}$, the result follows.

Table 2 summarizes some of the main formulas presented above.

\subsection{Obtaining the AQC from the DAQ}

To obtain an explicit expression for $\Sigma$ in terms of the dual absolute quadric $Q_{\infty}^{*}$ we apply (16) and (17) to the left-hand side of (35),

$\frac{1}{2} \operatorname{trace}\left(\left(\mathrm{L}^{* \prime}\right)^{\top} \mathrm{Q}_{\infty}^{*} \mathrm{~L}^{*} \mathrm{Q}_{\infty}^{*}\right)=\ell_{\mathrm{L}^{*}}^{\top} \ell_{\mathrm{Q}_{\infty}^{*} \mathrm{~L}^{*} \mathrm{Q}_{\infty}^{*}}=\ell_{\mathrm{L}^{\prime}}^{\top} \Omega \ell_{\mathrm{Q}_{\infty}^{*} \mathrm{~L}^{*} \mathrm{Q}_{\infty}^{*}}$

Comparing the right-hand sides of (35) and (40), we obtain that

$\Sigma \ell_{\mathrm{L}}=\Omega \ell_{\mathrm{Q}_{\infty}^{*} \mathrm{~L}^{*} \mathrm{Q}_{\infty}^{*}}$

By making $\ell_{\mathrm{L}}$ take the values of the canonical basis we can obtain explicit expressions for the columns of $\Sigma$. Thus, let $\mathbf{Q}_{\infty}^{*}=\left(\mathbf{q}_{1}, \mathbf{q}_{2}, \mathbf{q}_{3}, \mathbf{q}_{4}\right)$ and, according to (14), substitute $\mathrm{L}^{*}=\mathrm{M}\left(\mathbf{e}_{1}, \mathbf{e}_{2}\right)$ in the last equation to obtain the first column of $\Sigma$,

$$
\begin{aligned}
\Omega \ell_{\mathbf{Q}_{\infty}^{*} \mathrm{M}\left(\mathbf{e}_{1}, \mathbf{e}_{2}\right) \mathbf{Q}_{\infty}^{*}} & \stackrel{(11)}{=} \Omega \boldsymbol{\ell}_{\mathrm{M}\left(\mathbf{q}_{1}, \mathbf{q}_{2}\right)} \\
& \stackrel{(21)}{=} \Omega\left(\mathbf{q}_{1} \wedge \mathbf{q}_{2}\right) \\
& \stackrel{(22)}{=} \mathbf{q}_{1} \wedge \mathbf{q}_{2} .
\end{aligned}
$$

Proceeding analogously with the other columns, we conclude that the matrix $\Sigma$ is

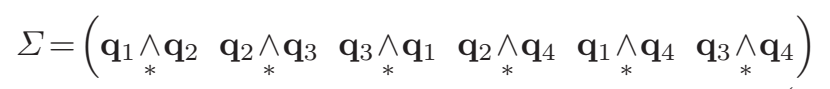

or, defining $\widetilde{\mathbf{Q}_{\infty}^{*}}$ as in (25) and using (22),

$\Sigma=\Omega \widetilde{Q_{\infty}^{*}} \Omega$

where we have used that right-multiplication by $\Omega$ inverts the order of the columns.

\subsection{The AQC in a Euclidean coordinate system}

Substituting the canonical form $\left(\mathbf{Q}_{\infty}^{*}\right)_{\text {euc }}=\left(\mathbf{e}_{1}, \mathbf{e}_{2}, \mathbf{e}_{3}, \mathbf{0}\right)$ in (43), we obtain $\Sigma=\Sigma_{\text {euc }}$ in homogeneous Euclidean coordinates $(X, Y, Z, T)^{\top}$, where

$\Sigma_{\text {euc }}=\left(\begin{array}{ll}\mathrm{I}_{3 \times 3} & 0_{3 \times 3} \\ 0_{3 \times 3} & 0_{3 \times 3}\end{array}\right)$. 
Table 2 Line representation, incidence and orthogonality in terms of Plücker matrices and coordinates.

\begin{tabular}{|c|c|c|}
\hline & Plücker matrices & Plücker coordinates \\
\hline Line obtained by & $\mathrm{L} \sim \mathrm{M}\left(\mathbf{X}_{1}, \mathbf{X}_{2}\right)$ & $\ell \sim \mathbf{X}_{1} \wedge \mathbf{X}_{2}$ \\
\hline join of two points & $\mathrm{L}^{*} \sim \mathrm{M}^{*}\left(\mathbf{X}_{1}, \mathbf{X}_{2}\right)$ & $\ell \sim \Omega\left(\mathbf{X}_{1} \bigwedge_{*} \mathbf{X}_{2}\right)$ \\
\hline Line obtained by & $\mathrm{L} \sim \mathrm{M}^{*}\left(\boldsymbol{\pi}_{1}, \boldsymbol{\pi}_{2}\right)$ & $\boldsymbol{\ell} \sim \boldsymbol{\pi}_{1} \wedge_{*} \boldsymbol{\pi}_{2}$ \\
\hline meet of two planes & $\mathrm{L}^{*} \sim \mathrm{M}\left(\boldsymbol{\pi}_{1}, \boldsymbol{\pi}_{2}\right)$ & $\boldsymbol{\ell} \sim \Omega\left(\boldsymbol{\pi}_{1} \wedge \boldsymbol{\pi}_{2}\right)$ \\
\hline Valid line & $\operatorname{trace}\left(\mathrm{LL}^{*}\right)=0=\operatorname{trace}\left(\mathrm{L}^{*} \mathrm{~L}\right)$ & $\boldsymbol{\ell}^{\top} \Omega \boldsymbol{\ell}=0$ \\
\hline Coplanar lines & $\operatorname{trace}\left(\mathrm{LL}^{* \prime}\right)=0=\operatorname{trace}\left(\mathrm{L}^{*} \mathrm{~L}^{\prime}\right)$ & $\ell^{\top} \Omega \ell^{\prime}=0$ \\
\hline Change of coordinates & $\mathrm{L}^{\prime} \sim \mathrm{HLH}^{\top}$ & $\ell^{\prime}=\widetilde{\mathrm{H}} \ell$ \\
\hline $\mathbf{X}^{\prime}=\mathrm{HX}, \quad \boldsymbol{\pi}^{\prime}=\mathrm{H}^{-\top} \boldsymbol{\pi}$ & $\mathrm{L}^{* \prime} \sim \mathrm{H}^{-\top} \mathrm{L}^{*} \mathrm{H}^{-1}$ & \\
\hline Orthogonal lines & $\operatorname{trace}\left(\mathrm{L}^{* \prime} \mathrm{Q}_{\infty}^{*} \mathrm{~L}^{*} \mathrm{Q}_{\infty}^{*}\right)=0$ & $\ell^{\top} \Sigma \ell^{\prime}=0$ \\
\hline Isotropic lines & $\operatorname{trace}\left[\left(\mathrm{L}^{*} \mathrm{Q}_{\infty}^{*}\right)^{2}\right]=0$ & $\ell^{\top} \Sigma \ell=0$ \\
\hline
\end{tabular}

Conversely, if $\Sigma \sim \Sigma_{\text {euc }}$ the coordinates must be Euclidean. In fact, the plane at infinity has equation $T=0$ since this is the plane in which the columns of $\Omega \Sigma$ lie. Now we can obtain the equation of the absolute conic by imposing that the line through the point $\mathbf{p}=(0,0,0,1)^{\top}$ and a point $\mathbf{q}=(X, Y, Z, 0)^{\top}$ belongs to the AQC. According to (21) this line has Plücker coordinates $\ell=\boldsymbol{\ell}_{\mathrm{M}(\mathbf{p}, \mathbf{q})}=(-Z,-X,-Y, 0,0,0)$, so that the condition is

$\ell^{\top} \Sigma_{\text {euc }} \ell=X^{2}+Y^{2}+Z^{2}=0$.

Therefore, the absolute conic has the canonical equations $X^{2}+Y^{2}+Z^{2}=T=0$ and thus the coordinate system is Euclidean.

\subsection{Changes of coordinates and the AQC}

Let $\mathbf{p}=\mathrm{H}^{\prime}$ be a coordinate change in $\mathbb{P}^{3}$ and $\boldsymbol{\ell}=\tilde{\mathrm{H}} \boldsymbol{\ell}^{\prime}$ the corresponding coordinate change between Plücker coordinates (25). The AQC being a quadric, its matrix changes according to the rule

$\Sigma^{\prime}=\tilde{\mathrm{H}}^{\top} \Sigma \tilde{\mathrm{H}}$.

From this, (25), (28), and (45) it follows that if $\mathbf{p}$ are Euclidean coordinates, the AQC in the non-Euclidean coordinate system can be written in terms of the rows $\mathbf{v}_{i}$ of $\mathrm{H}$ as

$$
\begin{aligned}
\Sigma^{\prime}= & \left(\mathbf{v}_{3} \wedge \mathbf{v}_{4} \quad \mathbf{v}_{1} \wedge \mathbf{v}_{4} \quad \mathbf{v}_{2} \wedge \mathbf{v}_{4}\right) \\
& \cdot\left(\mathbf{v}_{3} \wedge \mathbf{v}_{4} \quad \mathbf{v}_{1} \wedge \mathbf{v}_{4} \quad \mathbf{v}_{2} \wedge \mathbf{v}_{4}\right)^{\top}
\end{aligned}
$$

Alternatively, using

$\tilde{\mathrm{H}}=\operatorname{det}(\mathrm{H}) \widetilde{\Omega \mathrm{H}^{-\top}} \Omega$, that derives from (26) and (29), we can write $\Sigma^{\prime}$ in terms of the columns $\mathbf{u}_{i}$ of $\mathrm{H}^{-1}$ as

$$
\begin{aligned}
\Sigma^{\prime}= & \left(\begin{array}{lll}
\mathbf{u}_{1} \underset{*}{\wedge} \mathbf{u}_{2} & \mathbf{u}_{2} \underset{*}{\wedge} \mathbf{u}_{3} & \mathbf{u}_{3} \underset{*}{\wedge} \mathbf{u}_{1}
\end{array}\right) \\
& \cdot\left(\begin{array}{lll}
\mathbf{u}_{1} \underset{*}{\wedge} \mathbf{u}_{2} & \mathbf{u}_{2}{\underset{*}{\wedge}}_{\mathbf{u}_{3}} \mathbf{u}_{3}{\underset{*}{\wedge} \mathbf{u}_{1}}^{\top}
\end{array}\right)^{\top} .
\end{aligned}
$$

It is in this form that the matrix $\Sigma$ was introduced in [20, Lemma 3], where it was interpreted as the matrix establishing orthogonality between lines.

\subsection{A linear constraint on the AQC}

The coefficients of the AQC satisfy the linear constraint given by $\operatorname{trace}(\Omega \Sigma)=0$ :

$$
\begin{aligned}
\operatorname{trace}(\Omega \Sigma) \stackrel{(45,47)}{=} \operatorname{trace}\left(\Omega \tilde{\mathrm{H}}^{\top} \Sigma_{\text {euc }} \tilde{\mathrm{H}}\right) \\
=\operatorname{trace}\left(\tilde{\mathrm{H}} \Omega \tilde{\mathrm{H}}^{\top} \Sigma_{\text {euc }}\right) \\
\stackrel{(28)}{=} \operatorname{trace}\left(\widetilde{\mathrm{H}^{\top}} \widetilde{\mathrm{H}^{\top}} \Sigma_{\text {euc }}\right) \\
\stackrel{(26)}{=} \operatorname{trace}\left(\operatorname{det}\left(\mathrm{H}^{\top}\right) \Omega \Sigma_{\text {euc }}\right)=0 .
\end{aligned}
$$

Thus the AQC matrices are contained in a hyperplane of the vector space of the $6 \times 6$ symmetric matrices.

\subsection{Angle between two lines}

The angle $\theta \in[0, \pi / 2]$ between two real lines $\boldsymbol{\ell}$ and $\boldsymbol{\ell}^{\prime}$ is defined as $\min (\phi, \pi-\phi)$ where $\phi$ is the angle between any two direction vectors of the lines. Using Plücker coordinates, it can be computed in terms of $\Sigma$ as

$\cos \theta=\frac{\left|\ell^{\top} \Sigma \boldsymbol{\ell}^{\prime}\right|}{\sqrt{\left(\boldsymbol{\ell}^{\top} \Sigma \boldsymbol{\ell}\right)\left(\boldsymbol{\ell}^{\prime \top} \Sigma \boldsymbol{\ell}^{\prime}\right)}}$. 
It is enough to prove this formula for a Euclidean coordinate system. Any vector representative of the point of intersection of the line $\boldsymbol{\ell}$ with the plane at infinity $T=0$ is a direction vector of $\boldsymbol{\ell}$. We obtain from (5) and (15) that this point of intersection is

$$
\left(\begin{array}{cccc}
0 & l_{6} & -l_{4} & l_{2} \\
-l_{6} & 0 & l_{5} & l_{3} \\
l_{4} & -l_{5} & 0 & l_{1} \\
-l_{2} & -l_{3} & -l_{1} & 0
\end{array}\right)\left(\begin{array}{l}
0 \\
0 \\
0 \\
1
\end{array}\right)=\left(\begin{array}{l}
l_{2} \\
l_{3} \\
l_{1} \\
0
\end{array}\right)
$$

so that the formula for the angle between two lines given their direction vectors $\mathbf{d}=\left(l_{2}, l_{3}, l_{1}\right)^{\top}$ and $\mathbf{d}^{\prime}=$ $\left(l_{2}^{\prime}, l_{3}^{\prime}, l_{1}^{\prime}\right)^{\top}$ becomes (cf. [20])

$\cos \theta=\frac{\left|\mathbf{d}^{\top} \mathbf{d}^{\prime}\right|}{\sqrt{\left(\mathbf{d}^{\top} \mathbf{d}\right)\left(\mathbf{d}^{\prime \top} \mathbf{d}^{\prime}\right)}}=\frac{\left|\ell^{\top} \Sigma_{\mathrm{euc}} \ell^{\prime}\right|}{\sqrt{\left(\ell^{\top} \Sigma_{\mathrm{euc}} \ell\right)\left(\ell^{\prime \top} \Sigma_{\mathrm{euc}} \ell^{\prime}\right)}}$.

3.8 Computing the camera intrinsic parameters from $\Sigma$

The intrinsic parameter matrix $\mathrm{K}$ of a linear projective camera in a Euclidean coordinate system, $\mathrm{P}=\mathrm{K}(\mathrm{R} \mid-$ $\mathrm{Rt}$ ), is given by

$\mathrm{K}=\left(\begin{array}{ccc}\alpha_{u} & -\alpha_{u} \cot \theta & u_{0} \\ 0 & \alpha_{v} / \sin \theta & v_{0} \\ 0 & 0 & 1\end{array}\right)$

where $u_{0}$ and $v_{0}$ are the affine coordinates of the principal point, $\alpha_{u}$ and $\alpha_{v}$ are the pixel scale factors and $\theta$ is the skew angle between the axes of the pixel coordinates. We denote by $\tau=\alpha_{u} / \alpha_{v}$ the pixel aspect ratio. The matrix $\mathrm{R}$ is a rotation matrix which gives the camera orientation, and $\mathbf{t}$ are the coordinates of the camera centre.

\subsubsection{Image of the absolute conic and intrinsic parameter matrix}

The image of the absolute conic (IAC) given by a projection $P$ is the set of points of the image plane whose back-projected lines intersect the absolute conic (see Fig. 2). Thus its matrix $\omega$ can be derived from $\Sigma$ using (31) and (37) as

$\omega=\mathcal{P} \Sigma \mathcal{P}^{\top}$

with $\mathcal{P}$ given in (30). As is well known [11] the intrinsic parameter matrix can be retrieved from the IAC by Cholesky factorization from the relationship $\omega^{*}=\mathrm{KK}^{\top}$, where $\omega^{*} \sim \omega^{-1}$ is the dual of the IAC. Besides, some intrinsic parameters can be obtained explicitly, as we see in the following.

\subsubsection{Skew angle}

The skew angle can be computed as the one defined by the back-projected lines corresponding to the image points $(1,0,0)$ and $(0,1,0)$. Combining equations (31) and (50) we obtain the formula

$$
\begin{aligned}
\cos \theta & =\frac{\left|\omega_{12}\right|}{\sqrt{\overline{\omega_{11} \omega_{22}}}} \quad\left|\left(\boldsymbol{\pi}_{2} \wedge \boldsymbol{\pi}_{3}\right)^{\top} \Sigma\left(\boldsymbol{\pi}_{3} \wedge \boldsymbol{\pi}_{1}\right)\right| \\
& =\frac{\sqrt{\left[\left(\boldsymbol{\pi}_{2} \wedge \boldsymbol{\pi}_{3}\right)^{\top} \Sigma\left(\boldsymbol{\pi}_{2} \wedge \boldsymbol{\pi}_{3}\right)\right]\left[\left(\boldsymbol{\pi}_{3} \wedge \boldsymbol{\pi}_{1}\right)^{\top} \Sigma\left(\boldsymbol{\pi}_{3} \wedge \boldsymbol{\pi}_{1}\right)\right]}}{\sqrt{*}} .
\end{aligned}
$$

\subsubsection{Aspect ratio}

To compute the aspect ratio $\tau$ we observe that the image points of affine coordinates $(0,0),(\tau, 0),(0,1)$ and $(\tau, 1)$ are the vertices of a rhomb. Therefore the diagonal directions $(\tau, 1,0)$ and $(-\tau, 1,0)$ are orthogonal, and we have the relation

$\left(\begin{array}{lll}\tau & 1 & 0\end{array}\right) \mathcal{P} \Sigma \mathcal{P}^{\top}\left(\begin{array}{c}-\tau \\ 1 \\ 0\end{array}\right)=0$

from which we obtain

$\tau^{2}=\frac{\omega_{22}}{\omega_{11}}=\frac{\left(\boldsymbol{\pi}_{3} \wedge \boldsymbol{\pi}_{1}\right)^{\top} \Sigma\left(\boldsymbol{\pi}_{3} \bigwedge_{*} \boldsymbol{\pi}_{1}\right)}{\left(\boldsymbol{\pi}_{2} \wedge \boldsymbol{\pi}_{3}\right)^{\top} \Sigma\left(\boldsymbol{\pi}_{2} \bigwedge_{*} \boldsymbol{\pi}_{3}\right)}$.

Observe that the well-known conditions for the projection matrices of square-pixel cameras in Euclidean coordinates [9] are a particular case of (53) and (54) for $\theta=\pi / 2, \tau=1$, and $\Sigma=\Sigma_{\text {euc. }}$.

\subsubsection{Principal point}

The principal point $\mathbf{u}_{0}=\left(u_{0}, v_{0}, 1\right)^{\top}$ is the image point whose back-projected line is orthogonal to the image plane. Taking for instance the image plane directions $\mathbf{e}_{1}=(1,0,0)^{\top}$ and $\mathbf{e}_{2}=(0,1,0)^{\top}$, we have

$\mathbf{u}_{0}^{\top} \mathcal{P} \Sigma \mathcal{P}^{\top} \mathbf{e}_{i}=0, i=1,2$

leading to an explicit formula in terms of the cross product of two vectors in $\mathbf{C}^{3}$,

$$
\begin{aligned}
\mathbf{u}_{0} & \sim\left(\mathcal{P} \Sigma \mathcal{P}^{\top} \mathbf{e}_{1}\right) \times\left(\mathcal{P} \Sigma \mathcal{P}^{\top} \mathbf{e}_{2}\right) \\
& =\left(\begin{array}{c}
\omega_{12} \omega_{23}-\omega_{22} \omega_{13} \\
\omega_{12} \omega_{13}-\omega_{11} \omega_{23} \\
\omega_{11} \omega_{22}-\omega_{12}^{2}
\end{array}\right)
\end{aligned}
$$




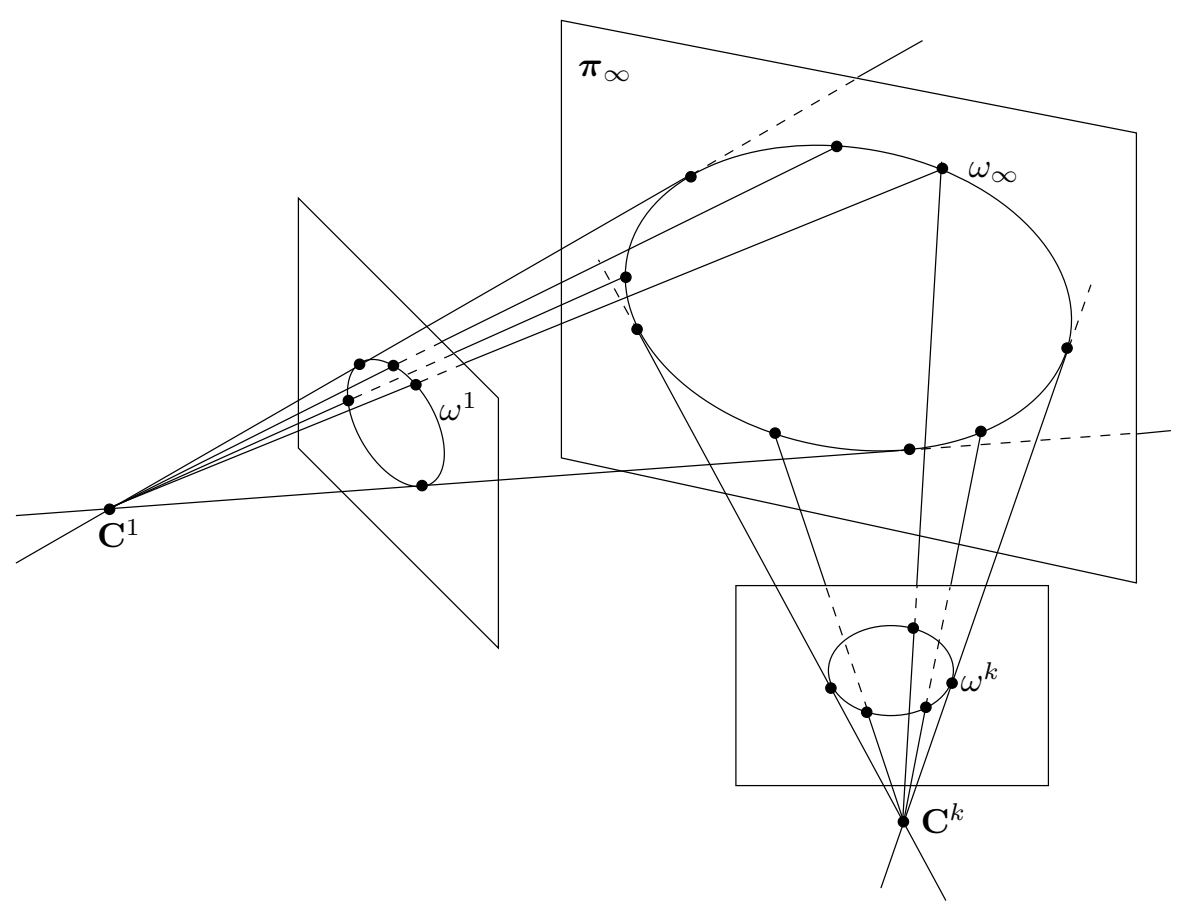

Fig. 2 Obtaining the IAC from the AQC. $\omega_{\infty}$ is the absolute conic.

\subsection{Computing the DAQ from the AQC}

Formula (43), giving the AQC matrix $\Sigma$ in terms of the DAQ matrix $\mathrm{Q}_{\infty}^{*}$, can be inverted by solving an homogeneous linear system of equations stemming from the following properties, that are immediate from (7):

$$
\begin{aligned}
\mathrm{M}^{*}\left(\mathbf{q}_{i}, \mathbf{q}_{j}\right) \mathbf{q}_{i} & =\mathbf{0} \\
\mathrm{M}^{*}\left(\mathbf{q}_{i}, \mathbf{q}_{j}\right) \mathbf{q}_{k} & =\mathrm{M}^{*}\left(\mathbf{q}_{k}, \mathbf{q}_{i}\right) \mathbf{q}_{j} .
\end{aligned}
$$

In our case the $\mathrm{M}^{*}$ matrices above can be built from the columns of $\Sigma$ using formulas (43) and (21), and the right-multiplying $\mathbf{q}_{l}$ are the unknowns. A solution is obtained within the linear space of dimension ten of the symmetric $4 \times 4$ matrices and then approximated by the closest rank-three symmetric matrix by annuling the smallest singular value [28, p. 35].

\subsection{Obtaining a Euclidean coordinate system from} the AQC

Let $\mathbf{Q}_{\infty}^{*}$ and $\left(\mathbf{Q}_{\infty}^{*}\right)_{\text {euc }}=\operatorname{diag}(1,1,1,0)$ be the matrices of the DAQ with respect to a projective and a Euclidean coordinate system, respectively. If $\mathrm{H}$ is any regular $4 \times 4$ matrix such that

$\mathbf{Q}_{\infty}^{*}=\mathrm{H}\left(\mathbf{Q}_{\infty}^{*}\right)_{\mathrm{euc}} \mathrm{H}^{\top}$

then $\mathrm{H}$ is indeed a matrix changing from a Euclidean coordinate system to the projective coordinate system in which $Q_{\infty}^{*}$ is expressed (see [11, p. 447]). A practical way to find such a factorization is to compute a SVD of the positive semidefinite matrix $Q_{\infty}^{*}$,

$\mathbf{Q}_{\infty}^{*}=\mathrm{U} \operatorname{diag}\left(\sigma_{1}, \sigma_{2}, \sigma_{3}, 0\right) \mathbf{U}^{\top}$,

and define $\mathrm{H}$ such that equation (57) holds true, e.g.

$\mathrm{H}=\mathrm{U} \operatorname{diag}\left(\sqrt{\sigma_{1}}, \sqrt{\sigma_{2}}, \sqrt{\sigma_{3}}, 1\right)$.

However, if we are given the matrix $\Sigma$ corresponding to an arbitrary coordinate system of $\mathbb{P}^{3}$ and a factorization

$\Sigma=\mathrm{G}^{\top} \Sigma_{\text {euc }} \mathrm{G}$

for a regular matrix G, this matrix is not necessarily of the form $\widetilde{\mathrm{H}}$ for any regular matrix $\mathrm{H}$. To check this, observe that the last three columns of $\mathrm{G}^{\top}$ are not determined by (60), and this freedom is not compatible with relation (26). In fact, equation (60) determines matrix $\mathrm{G}$ up to left-multiplication by a matrix of the form $\left(\begin{array}{ll}\mathrm{U} & 0 \\ \mathrm{C} & \mathrm{D}\end{array}\right)$, where $\mathrm{U}$ is a $3 \times 3$ orthogonal matrix and C, D are arbitrary $3 \times 3$ matrices with $\operatorname{det} \mathrm{D} \neq 0$ (see Sect. (A.6)). Nevertheless, the factorization (60) does provide the matrix of the change of coordinates to a Euclidean reference, according to the following theorem (proved in Sect. A.7), that formalizes the technique proposed in [20]. 
Table 3 Comparison between the DAQ and the AQC.

\begin{tabular}{|c|c|c|}
\hline & $\begin{array}{l}\text { Dual Absolute Quadric } \\
\text { DAQ }\end{array}$ & $\begin{array}{l}\text { Absolute Quadratic Complex } \\
\text { AQC }\end{array}$ \\
\hline Matrix symbol & $\mathrm{Q}_{\infty}^{*}$ & $\Sigma=\Omega \widetilde{\mathrm{Q}_{\infty}^{*}} \Omega$ \\
\hline Orthogonality of... & planes: $\boldsymbol{\pi}^{\top} \mathbf{Q}_{\infty}^{*} \boldsymbol{\pi}^{\prime}=0$ & lines: $\boldsymbol{\ell}^{\top} \Sigma \boldsymbol{\ell}^{\prime}=0$ \\
\hline Constraints & $\begin{array}{l}\operatorname{det}\left(\mathbf{Q}_{\infty}^{*}\right)=0 \\
\operatorname{rank}\left(\mathbf{Q}_{\infty}^{*}\right)=3\end{array}$ & $\begin{array}{l}\Sigma \Omega \Sigma=0, \quad \operatorname{trace}(\Omega \Sigma)=0 \\
\operatorname{rank}(\Sigma)=3\end{array}$ \\
\hline & $\mathrm{Q}_{\infty}^{*}$ is positive semidefinite & $\Sigma$ is positive semidefinite \\
\hline Kernel & $\begin{array}{l}\operatorname{ker} \mathbf{Q}_{\infty}^{*}=\boldsymbol{\pi}_{\infty} \\
\mathbf{Q}_{\infty}^{*} \boldsymbol{\pi}_{\infty}=\mathbf{0}\end{array}$ & $\begin{array}{l}\operatorname{ker} \Sigma=\boldsymbol{\beta}_{\infty} \\
\text { (set of all the lines in } \boldsymbol{\pi}_{\infty} \text { ) }\end{array}$ \\
\hline Camera representation & $\mathrm{P}=\left(\boldsymbol{\pi}_{1}, \boldsymbol{\pi}_{2}, \boldsymbol{\pi}_{3}\right)^{\top}$ & $\begin{array}{l}\mathcal{P}=\left(\boldsymbol{\ell}_{1}, \boldsymbol{\ell}_{2}, \boldsymbol{\ell}_{3}\right)^{\top} \\
=\left(\boldsymbol{\pi}_{2} \wedge \boldsymbol{\pi}_{3}, \boldsymbol{\pi}_{3} \wedge \boldsymbol{\pi}_{1}, \boldsymbol{\pi}_{1} \wedge \boldsymbol{\pi}_{2}\right)^{\top}\end{array}$ \\
\hline Projection eq. & $\omega^{*} \sim \mathrm{PQ}_{\infty}^{*} \mathrm{P}^{\top}$ & $\omega \sim \mathcal{P} \Sigma \mathcal{P}^{\top}$ \\
\hline Upgrading matrix & $\mathrm{H}=\left(\mathbf{h}_{1}, \mathbf{h}_{2}, \mathbf{h}_{3}, \mathbf{h}_{4}\right)$ & $\mathcal{R}=\left(\mathbf{r}_{1}, \mathbf{r}_{2}, \mathbf{r}_{3}\right)$ \\
\hline $\mathbf{X}_{\mathrm{euc}}=\mathrm{H}^{-1} \mathbf{X}$ & $\mathrm{H}_{123}=\left(\mathbf{h}_{1}, \mathbf{h}_{2}, \mathbf{h}_{3}\right)$ & 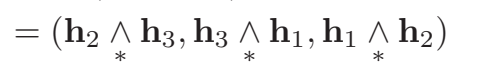 \\
\hline Factorization or & $\mathrm{Q}_{\infty}^{*}=\mathrm{H}_{123} \mathrm{H}_{123}^{\top}$ & $\Sigma=\mathcal{R}^{*} \mathcal{R}^{\top}{ }^{*}$ \\
\hline change of coords. & $\mathrm{Q}_{\infty}^{*}=\mathrm{H}\left(\mathrm{Q}_{\infty}^{*}\right)_{\mathrm{euc}} \mathrm{H}^{\top}$ & $\Sigma=\Omega \widetilde{\mathrm{H}} \Omega \Sigma_{\text {euc }} \Omega \widetilde{\mathrm{H}}^{\top} \Omega$ \\
\hline Euclidean form & $\left(Q_{\infty}^{*}\right)_{\mathrm{euc}}=\operatorname{diag}(1,1,1,0)$ & $\Sigma_{\text {euc }}=\operatorname{diag}(1,1,1,0,0,0)$ \\
\hline
\end{tabular}

Theorem 1 We consider a factorization of the $A Q C$ matrix of the form $\Sigma=\mathrm{G}^{\top} \Sigma_{\text {euc }} \mathbf{G}$ with $\mathrm{G}^{\top}=\left(\mathbf{r}_{1}, \ldots, \mathbf{r}_{6}\right)$. Then the vectors $\mathbf{r}_{i}, i=1,2,3$, can be written as $\mathbf{r}_{1}=$ $\mathbf{v}_{3} \wedge \mathbf{v}_{4}, \quad \mathbf{r}_{2}=\mathbf{v}_{1} \wedge \mathbf{v}_{4}, \quad \mathbf{r}_{3}=\mathbf{v}_{2} \wedge \mathbf{v}_{4}$ for some linearly independent vectors $\mathbf{v}_{i}$ such that the matrix $\mathrm{H}^{\top}=$ $\left(\mathbf{v}_{1}, \mathbf{v}_{2}, \mathbf{v}_{3}, \mathbf{v}_{4}\right)$ provides a coordinate change from the current coordinate system to a Euclidean one, so that points and planes transform as $\mathbf{X}_{\mathrm{euc}}=\mathrm{H} \mathbf{X}$ and $\boldsymbol{\pi}_{\mathrm{euc}}=$ $\mathrm{H}^{-\top} \boldsymbol{\pi}$, respectively.

Therefore the vectors $\mathbf{v}_{i}$ are the coordinates of the faces of a Euclidean coordinate tetrahedron. In particular, $\mathbf{v}_{4}$ is the plane at infinity. Hence the Plücker vectors $\Omega \mathbf{r}_{1}=\mathbf{v}_{3} \wedge \mathbf{v}_{4}, \Omega \mathbf{r}_{2}=\mathbf{v}_{1} \wedge \mathbf{v}_{4}, \Omega \mathbf{r}_{3}=\mathbf{v}_{2} \wedge \mathbf{v}_{4}$ represent the three lines of the plane at infinity of the Euclidean coordinate tetrahedron.

Observe that the decomposition (60) can be obtained by SVD followed by setting to zero the three lowest singular values. The vectors $\mathbf{v}_{i}$ can be computed from the $\mathbf{r}_{i}$ as follows. We first obtain the L-matrices $\mathrm{M}_{k l}=$ $\mathrm{M}\left(\mathbf{v}_{k}, \mathbf{v}_{l}\right)$ of the lines $\mathbf{r}_{i}$ by the conditions $\mathbf{r}_{1}=\boldsymbol{\ell}_{\mathrm{M}_{34}}$, $\mathbf{r}_{2}=\boldsymbol{\ell}_{\mathrm{M}_{14}}$ and $\mathbf{r}_{3}=\boldsymbol{\ell}_{\mathrm{M}_{24}}$. Then we find $\mathbf{v}_{4}$ as a common vector in the kernel of the associated $\mathrm{L}^{*}$-matrices $\mathrm{M}_{i 4}^{*}$, $i=1,2,3$. Finally, substitute the value obtained for $\mathbf{v}_{4}$ in the conditions $\mathbf{r}_{1}=\boldsymbol{\ell}_{\mathrm{M}\left(\mathbf{v}_{3}, \mathbf{v}_{4}\right)}, \mathbf{r}_{2}=\boldsymbol{\ell}_{\mathrm{M}\left(\mathbf{v}_{1}, \mathbf{v}_{4}\right)}$ and $\mathbf{r}_{3}=\boldsymbol{\ell}_{\mathrm{M}\left(\mathbf{v}_{2}, \mathbf{v}_{4}\right)}$, to obtain the vectors $\mathbf{v}_{i}, i=1,2,3$, by solving three linear systems of equations.
Some of the useful formulas that explain the similarities between the DAQ and the AQC are summarized in Table 3.

\subsection{Characterization of the AQC matrices}

The following theorem provides a characterization of the AQC matrices.

Theorem $2 A$ real $6 \times 6$ symmetric matrix $\Sigma$ is the matrix of the $A Q C$ in some coordinate system if and only if the following conditions hold true:

1. $\Sigma$ is rank-three and positive semidefinite.

2. $\Sigma \Omega \Sigma=0$.

3. $\operatorname{ker} \Sigma$ is the set of Plücker coordinates of the lines of a plane.

The proof of this result is given in Sect. A.8. The third condition seems to be very restrictive, but in fact the first and second conditions imply that $\operatorname{ker} \Sigma$ is either the star of lines through a point or the set of lines of a plane, and therefore this happens to be a dichotomic analysis, which can be verified using the formulas in Table 1. 


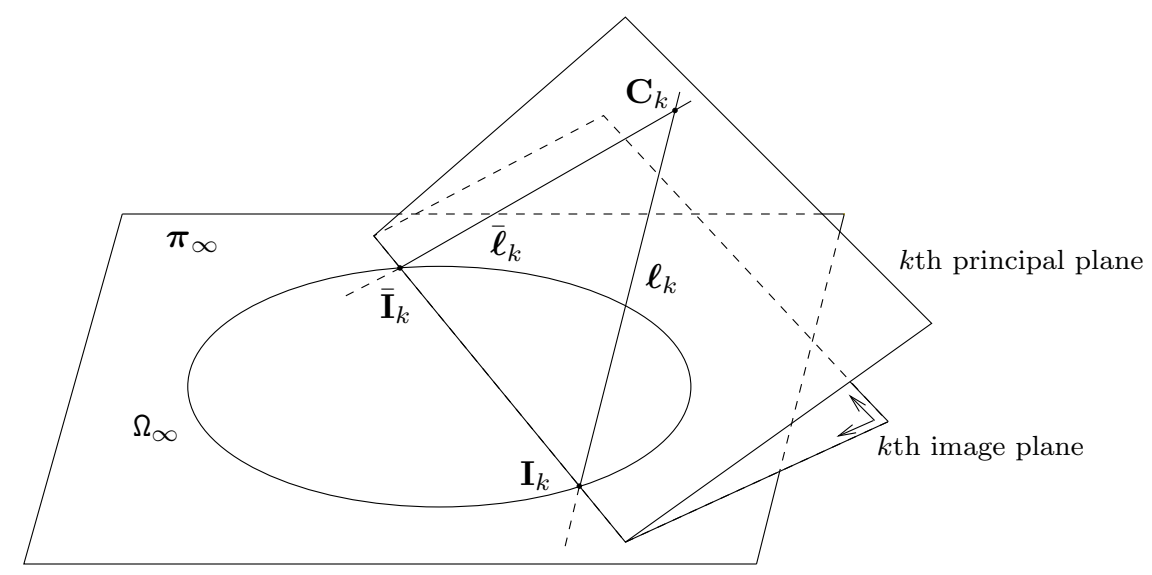

Fig. 3 Intersections with the absolute conic of the isotropic lines of the camera $k$, with center $\mathbf{C}_{k}$.

\section{The absolute quadratic complex and cameras with known pixel shape}

We will assume that the camera can be modeled [11] by the usual linear equation $\mathbf{X} \sim \mathrm{PX}$, where $\sim$ means equality up to a non-zero scale factor, $\mathbf{X}=(X, Y, Z, T)^{\top}$ denotes the homogeneous coordinates of a spatial point, $\mathbf{x}=(u, v, w)^{\top}$ represents the homogeneous coordinates of an image point, and $\mathrm{P}$ is the $3 \times 4$ matrix $\mathrm{P}=$ $\mathrm{K}(\mathrm{R} \mid-\mathrm{Rt})$, with $\mathrm{K}$ as in (51).

As is well known [11], it is possible to obtain a projective calibration only from point correspondences within two or more images. This means that, given a set of projected points $\mathbf{x}_{i j}$ obtained with $m$ cameras, $m \geq 2$, we can obtain a set of estimated matrices $\hat{\mathrm{P}}_{i}$ and point coordinates $\hat{\mathbf{X}}_{j}$ such that $\hat{\mathbf{x}}_{i j} \sim \hat{\mathrm{P}}_{i} \hat{\mathbf{X}}_{j}$ are equal to the observed $\mathbf{x}_{i j}$, where $\left\{\hat{\mathrm{P}}_{i}, \hat{\mathbf{X}}_{j}\right\}$ are equal to the Euclidean values $\left\{\mathrm{P}_{i}, \mathbf{X}_{j}\right\}$ up to a $4 \times 4$ non-singular matrix H, i.e., $\mathrm{P}_{i}=\hat{\mathrm{P}}_{i} \mathrm{H}$ and $\mathbf{X}_{j}=\mathrm{H}^{-1} \hat{\mathbf{X}}_{j}$.

Euclidean calibration can be defined as the obtainment of a matrix $\mathrm{H}^{-1}$ changing the projective coordinates of a given projective calibration to some Euclidean coordinate system, i.e., one in which the absolute conic has equations $X^{2}+Y^{2}+Z^{2}=T=0[25]$.

If the camera aspect ratio and skew are known, an affine coordinate transformation in the image permits to assume that the intrinsic parameter matrix has the form

$\mathrm{K}=\left(\begin{array}{ccc}\alpha & 0 & u_{0} \\ 0 & \alpha & v_{0} \\ 0 & 0 & 1\end{array}\right)$.

Let us consider the back-projected lines of image points $\mathbf{I}=(1, i, 0)^{\top}$ and $\overline{\mathbf{I}}=(1,-i, 0)^{\top}$. We will call them the isotropic lines of the camera (see [4, p. 184] for the motivation of the name). These lines intersect the absolute conic (Fig. 3). Indeed, if $\mathbf{X}=(X, Y, Z, 0)^{\top}$ is the intersection of one of these two lines with the plane at infinity, we have

$$
(1, \pm i, 0)^{\top} \sim \mathrm{PX}=\mathrm{KR}(X, Y, Z)^{\top},
$$

so that

$$
(X, Y, Z)^{\top} \sim \mathrm{R}^{\top} \mathrm{K}^{-1}(1, \pm i, 0)^{\top},
$$

and then

$$
\begin{aligned}
X^{2}+Y^{2}+Z^{2} & =(X, Y, Z)(X, Y, Z)^{\top} \\
& =(1, \pm i, 0) \mathrm{K}^{-\top} \mathrm{RR}^{\top} \mathrm{K}^{-1}(1, \pm i, 0)^{\top} \\
& =(1 \pm i)\left(\begin{array}{cc}
\alpha^{-2} & 0 \\
0 & \alpha^{-2}
\end{array}\right)\left(\begin{array}{c}
1 \\
\pm i
\end{array}\right)=0 .
\end{aligned}
$$

According to equation (31), one of the isotropic lines has Plücker coordinates

$\boldsymbol{\ell}=\mathcal{P}^{\top}(1, i, 0)^{\top}=\boldsymbol{\pi}_{2}{\underset{*}{*}}_{\boldsymbol{\pi}_{3}}+i \boldsymbol{\pi}_{3} \wedge \boldsymbol{\pi}_{1}$

the other one being its complex conjugate, so that we have the relationship

$$
\begin{aligned}
& \left(\boldsymbol{\pi}_{2} \wedge_{*} \boldsymbol{\pi}_{3}+i \boldsymbol{\pi}_{3} \wedge \boldsymbol{\pi}_{1}\right)^{\top} \Sigma\left(\boldsymbol{\pi}_{2}{ }_{*}^{\wedge} \boldsymbol{\pi}_{3}+i \boldsymbol{\pi}_{3} \wedge_{*}^{\wedge} \boldsymbol{\pi}_{1}\right)
\end{aligned}
$$

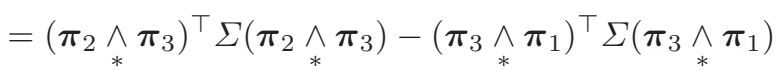

$$
\begin{aligned}
& +2 i\left(\boldsymbol{\pi}_{3}{\underset{*}{*}}_{\boldsymbol{\pi}_{1}}\right)^{\top} \Sigma\left(\boldsymbol{\pi}_{2}{\underset{*}{*}}_{3}\right)=0
\end{aligned}
$$

Observe that the vanishing of the real and imaginary parts of this expression are in fact equivalent, respectively, to having aspect ratio $\tau=1$ and skew angle $\theta=\pi / 2$, as follows from expressions (53) and (54).

Note that since Euclidean calibration amounts to determining eight parameters, we need eight equations as those provided by four square pixel cameras to obtain a discrete number of solutions. An additional fifth camera would be necessary to have a unique solution. The AQC provides a way to address this problem by means of linear equations with the drawback of having to use a larger number of cameras. 


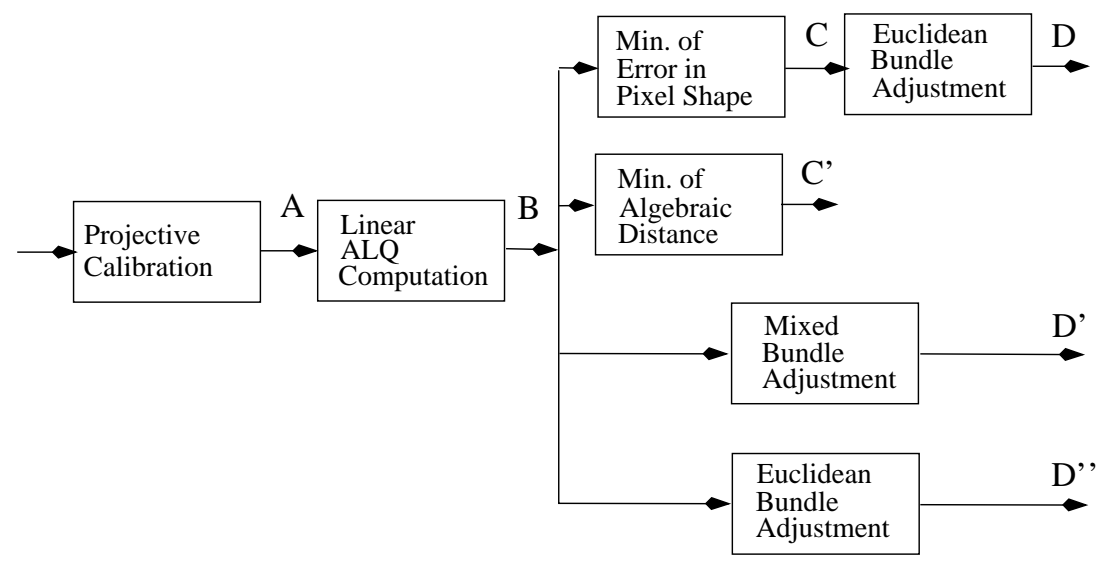

Fig. 4 Block diagram of the tested algorithms.

\section{Algorithms and experimental results}

The properties of the AQC suggest different strategies for the recovery of the Euclidean structure of space in a projective reconstruction obtained with square pixel cameras. As each camera provides a pair of linear equations (real and imaginary parts of (63)) in the coefficients of the $\mathrm{AQC}$, ten cameras provide 20 linear equations which, together with the linear constraint given in Sect. 3.6, permit to compute $\Sigma$. Then the rectifying homography H can be obtained from $\Sigma$ using theorem 1 . This technique, introduced in [20] and [30] has the advantage of providing a unique solution and the disadvantage of a potentially dangerous noise sensitivity.

But the theory of the AQC also provides us with cost functions that we can try to minimize with respect to the Euclidean upgrading matrix $\mathrm{H}$ employing a suitable parametrization of $\Sigma$. A technique of this type was first proposed in [20], considering then only the restriction associated with cameras with rectangular pixels $(\theta=\pi / 2)$. An alternative approach consists in the minimization of a cost function in terms of $\Sigma$, imposing on it the quadratic constraints (39). In [32] an algorithm is proposed that addresses this constrained optimization problem employing Sequential Quadratic Programming.

Observe that the non-linear refinement requires a minimum of five cameras instead of the ten cameras required by the linear algorithms. This is because four square pixel cameras provide eight non-linear equations in terms of $\mathrm{H}$, and eight is the number of parameters on which the unknown absolute conic depends. Thus four cameras provide a finite number of solutions, and five cameras yield in general a single solution. Although, as we will see, non-linear algorithms can be less sensitive to noise, they depend on the use of another algorithm for their initialization with an approximate solution.
In this section we present new non-linear autocalibration algorithms based on the AQC. To evaluate them rigorously we have divided the processing chain into basic building blocks, which have been exhaustively tested on synthetic data. Additionally, results on 3D reconstruction with real images are provided at the end of the section.

\subsection{Description of the algorithms}

The tested algorithms are summarized in Fig. 4. The processing starts with the block Projective Calibration, which is performed in two steps. The first one implements the fundamental matrix Gold Standard algorithm [11, p. 268] applying it to two of the cameras. The other cameras are calibrated from these using resectioning $[11$, p. 166]. This projective calibration is then improved using a projective factorization [11, p. 430].

Once obtained the projective calibration, we proceed to compute an Euclidean upgrading using the Linear AQC Computation given in Sect. 4. With this initial Euclidean upgrading, we perform a thorough testing of different possibilities for the improvement of the initial result, namely:

1. Perform directly Euclidean Bundle Adjustment using as starting point the Euclidean reconstruction provided by the linear algorithm. Euclidean bundle adjustment aims at minimizing the reprojection error

$$
\begin{gathered}
g\left(\mathrm{~K}_{i}, \mathrm{R}_{i}, \mathbf{t}_{i}, \mathbf{X}_{j}\right)=\sum_{i, j=1}^{m, n} d\left(\mathrm{P}_{i} \mathbf{X}_{j}, \mathbf{x}_{i j}\right)^{2}, \\
\mathrm{P}_{i}=\mathrm{K}_{i}\left(\mathrm{R}_{i} \mid-\mathrm{R}_{i} \mathbf{t}_{i}\right),
\end{gathered}
$$

where square-pixel conditions are enforced on the intrinsic parameter matrices $\mathrm{K}_{i}$. 
2. Apply first nonlinear optimization to improve the initial estimation of the AQC and then perform Euclidean bundle adjustment. Two possible non-linear cost functions have been tested. The first one consists in minimizing

$$
g(\mathrm{H})=\sum_{i=1}^{m}\left(\epsilon_{\theta}^{i}(\mathrm{H})^{2}+\epsilon_{\tau}^{i}(\mathrm{H})^{2}\right),
$$

where $\epsilon_{\theta}^{i}(\mathrm{H})=1-\theta\left(\hat{\mathrm{P}}_{i}, \Sigma(\mathrm{H})\right) / \theta_{i}$, and $\epsilon_{\tau}^{i}(\mathrm{H})=1-$ $\tau\left(\hat{\mathrm{P}}_{i}, \Sigma(\mathrm{H})\right) / \tau_{i}$ are, respectively, the relative errors in the $\theta$ and $\tau$ parameters for camera $i, \theta_{i}$ is the known skew angle and $\tau_{i}$ the known aspect ratio for camera $i$. Functions $\theta$ and $\tau$ are obtained from formulas (53) and (54). We compute $\Sigma(\mathrm{H})$ according to (44) and (57). This corresponds to Minimization of Error in Pixel Shape in Fig. 4. A Euclidean bundle adjustment is applied afterwards.

The other cost function we have considered is

$g(\mathrm{H})=\sum_{k=1}^{m}\left|\frac{\ell_{k}^{\top} \Sigma(\mathrm{H}) \ell_{k}}{\left\|\ell_{k}\right\|^{2}\|\Sigma(\mathrm{H})\|_{F}}\right|^{2}$,

where $\boldsymbol{\ell}_{k}$ is one of the isotropic lines of the $k$-th camera. This corresponds to Minimization of Algebraic Distance in Fig. 4. In both cases, the Levenberg -Marquardt algorithm is used in the optimization.

3. Alternatively, perform a modified version of projective bundle adjustment including a penalty term to enforce square-pixel cameras. This is called Mixed Bundle Adjustment in Fig. 4, and consists in minimizing the cost function

$$
\begin{aligned}
g\left(\mathrm{P}_{i}, \mathbf{X}_{j}, \mathrm{H}\right)= & \sum_{i, j=1}^{m, n} d\left(\mathrm{P}_{i} \mathbf{X}_{j}, \mathbf{x}_{i j}\right)^{2} \\
& +\xi\left(\sum_{i=1}^{m} \epsilon_{\theta}^{i}\left(\mathrm{P}_{i}, \mathrm{H}\right)^{2}+\epsilon_{\tau}^{i}\left(\mathrm{P}_{i}, \mathrm{H}\right)^{2}\right),
\end{aligned}
$$

where $\xi$ is a weighting factor that we set as $\xi=n^{2}$, $\epsilon_{\theta}^{i}\left(\mathrm{P}_{i}, \mathrm{H}\right)=1-\theta\left(\mathrm{P}_{i}, \Sigma(\mathrm{H})\right) / \theta_{i}$ and $\epsilon_{\tau}^{i}\left(\mathrm{P}_{i}, \mathrm{H}\right)=1-$ $\tau\left(\mathrm{P}_{i}, \Sigma(\mathrm{H})\right) / \tau_{i}$, with functions $\theta$ and $\tau$ deriving from (53) and (54). Note that the cost function $g\left(\mathrm{P}_{i}, \mathbf{X}_{j}, \mathrm{H}\right)$ is overparametrized, since the Euclidean variables $\mathrm{P}_{i}^{\prime}=\mathrm{P}_{i} \mathrm{H}$ and $\mathbf{X}_{j}^{\prime}=\mathrm{H}^{-1} \mathbf{X}_{j}$ should suffice. However, the overparametrization has been found to produce slightly better numerical results.

Non-linear optimization has been implemented using the standard Levenberg-Marquardt algorithm as described in [23]. Convergence criterion is given by the bounds for the maximum number of iterations (50) and for the value of the Levenberg-Marquardt explorationexploitation parameter lambda $\left(<10^{5}\right)$.

Sparse implementation of the Levenberg-Marquardt bundle adjustment has been used.

\subsection{Experiments with synthetic data}

The scheme has been tested with synthetic data in a series of experiments involving the reconstruction of a set of 100 points from their projections in 10 to $40 \mathrm{im}-$ ages taken with cameras with varying parameters. The $3 \mathrm{D}$ points lie close to the origin of coordinates of a Euclidean reference and the cameras are located at random positions lying approximately over a sphere centered at the origin and roughly pointing towards it, so that the set of projected points is approximately centered in the virtual CCD. Skew angle and aspect ratio are fixed at respective values $\pi / 2$ and 1 . Normalized focal length $\alpha$ is selected in each experiment at random with a uniform distribution centered at 2000 pixels with a maximum deviation of $\pm 10 \%$ from this value. The principal point is obtained from a uniform distribution with support in the square $[ \pm 400, \pm 300]$, to simulate a large variation. With these parameters the projected point coordinates have values within the range of an image of $1000 \times 750$ pixels and, in each image, the points are contained inside a square of side 500 pixels.

For each camera configuration, Gaussian noise with standard deviation $\sigma$ between 0 and 5 pixels is added to the projected point coordinates. This is the input of the algorithms in Fig. 4.

We compare the results of the algorithms in terms of reprojection error, error in the estimation of the camera intrinsic parameters, and computational cost. We first discuss the results for 15 cameras, shown in Fig. 5. The effect of varying the number of cameras will be analyzed later.

Our experiments also included the use of Euclidean Bundle Adjustment after Minimization of Algebraic Distance (C' in Fig. 4) with results indistinguishable from those of node $\mathrm{D}$. Therefore, they have been included in order to make the graphics more readable.

We first study the residual reprojection error (topleft graph and table in Fig. 4), including also the data for projective bundle adjustment. As is well known, there is a lower bound of this error [11, p.121] given by $\epsilon^{2} / \sigma^{2}=1-d / N$ where $N$ is the number of measurements and $d$ is the number of parameters on which the solution depends. So in the case of a projective reconstruction we have $N=2 m n$, where $n$ is the number of points and $m$ the number of images, and $d=$ $3 n+11 m-15$, since we have three parameters for each 3D point, eleven parameters for each projection matrix and we have to subtract 15 parameters to account for the projective world frame ambiguity. The case of a Euclidean reconstruction with square-pixel cameras is analogous except for the number of parameters of each 

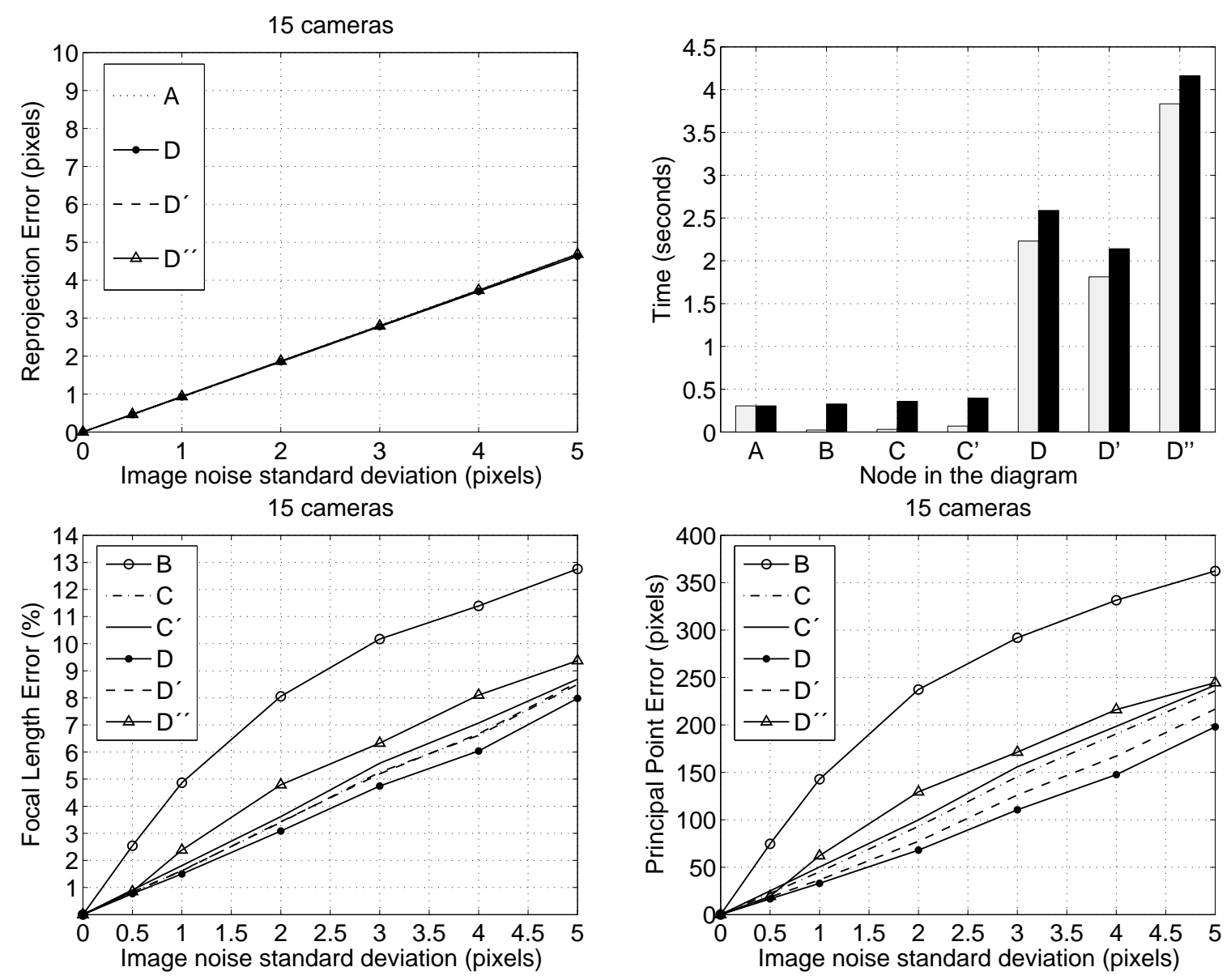

\begin{tabular}{|l|l|}
\hline Projective BA (theoretical bound) & 4.6097 \\
\hline Euclidean BA (theoretical bound) & 4.6296 \\
\hline Projective BA (experimental) & 4.6113 \\
\hline A & 4.7037 \\
\hline D & 4.6323 \\
\hline D' & 4.6360 \\
\hline D" & 4.6866 \\
\hline
\end{tabular}

Fig. 5 Results for 15 cameras. BA stands for bundle adjustment. Top left: Average residual reprojection error of the tested algorithms. Numbers refer to the nodes in the block diagram of Fig. 4. Top right: Cumulated computation time required to reach each node (black) and computation time spent in the block previous to the node (light gray). Middle left: Error in focal length estimation. Middle right: Error in principal point estimation. Bottom: Reprojection errors for noise $\sigma=5$.

projection matrix, that is 9 , and the number of ambiguous parameters of the world frame, which is 7 (a similarity transformation). Therefore

$$
\begin{aligned}
\epsilon_{\text {proj }}^{2} / \sigma^{2} & =1-(3 n+11 m-15) /(2 m n) \\
\epsilon_{\text {euc }}^{2} / \sigma^{2} & =1-(3 n+9 m-7) /(2 m n)
\end{aligned}
$$

in the cases of projective reconstruction and Euclidean reconstruction, respectively. In our experiments, the residual reprojection errors of all the considered autocalibration algorithms are nearly optimal.

Regarding the comparison of the computational costs, the direct use of Euclidean Bundle Adjustment right after the Linear AQC computation (node D") represents the worst case. Of the other two Euclidean reconstruction algorithms (D and D'), Mixed Bundle Adjustment is computationally the most efficient.

Now we compare the errors in the estimation of the camera intrinsic parameters. First we observe that, as expected, there is a noticeable improvement if any of the non-linear optimization techniques is included after Linear AQC Computation (node B). Among them, Minimization of Error in Pixel Shape plus Euclidean Bundle Adjustment (node D) provides the best results, while the direct use of Euclidean Bundle Ad- 

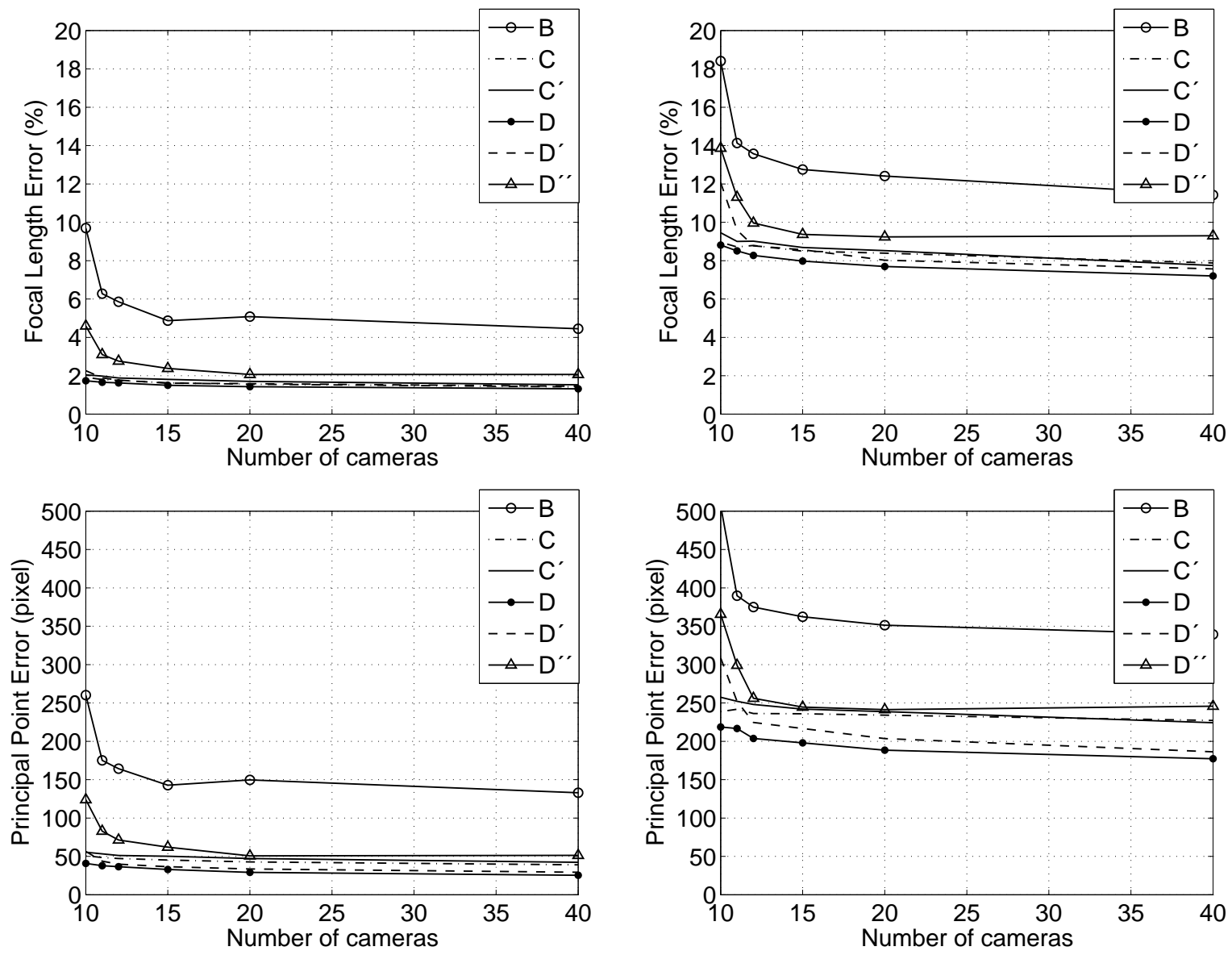

Fig. 6 Errors in the estimation of the focal length (top) and principal point (bottom) as a function of the number of cameras. Left: $\sigma=1$. Right: $\sigma=5$.

justment (node D") is the worst option in spite of showing, as mentioned before, the highest computational cost. On the other hand, Mixed Bundle Adjustment (node D') provides results very close to those of the optimal technique with noticeably computational saving and thus is our option of choice.

Figure 6 shows the influence of the number of cameras in the focal length and principal point errors, showing an early saturation effect. The computation time is not shown, as it is approximately proportional to the number of cameras. From these curves we observe that there is a meaningful improvement of the results when the number of cameras increases from 10 to 15 , but the improvement is marginal beyond this point.

\subsection{Experiments with real images}

In this section we present the experimental results of our algorithms tested on three real datasets: Checkerboard, Books, and Kings' Courtyard. The first dataset includes three checkerboard patterns to estimate di- rectly the intrinsic parameters of the cameras in order to compare them to the results of our algorithms. Table 4 shows some parameters of the data sets. The images and VRML reconstructions are available in http://www.gti.ssr.upm.es/ ${ }^{\sim}$ jir/comp_vis/AQC.

Table 4 Parameters of the experiments with real data.

\begin{tabular}{lccc}
\hline & $\begin{array}{c}\text { Checker- } \\
\text { board }\end{array}$ & Books & $\begin{array}{c}\text { Kings' } \\
\text { Courtyard }\end{array}$ \\
\hline Image size (pixels) & $1280 \times 960$ & $640 \times 480$ & $1024 \times 768$ \\
Total images & 25 & 18 & 23 \\
Points matched & 283 & 76 & 443 \\
Avg. visible points & 234 & 56 & 372 \\
\hline
\end{tabular}

For the Checkerboard dataset, 25 images of size 1280× 960 pixels were acquired with a digital camera. For the first 17 images, an equivalent focal length (in a $35 \mathrm{~mm}$ film) of $50 \mathrm{~mm}$ was selected, while for the last 8 images the focal lenght was doubled to $100 \mathrm{~mm}$. Note that variations due to auto-focus could not be controlled. 
Table 5 Reprojection errors in pixels for the experiments with real data.

\begin{tabular}{llll}
\hline Algorithm & Checkerboard & Books & Kings' Courtyard \\
\hline Gold Standard & 0.518 & 1.05 & 1.30 \\
Projective BA (first iterations) & $0.330,0.320,0.317$ & $0.592,0.561,0.553$ & $0.529,0.505,0.498$ \\
Projective BA (final) & 0.313 & 0.55 & 0.48 \\
Eucl. BA (node D) & 0.316 & 0.56 & 0.49 \\
Mixed BA & 0.318 & 0.55 & 0.49 \\
Estimated noise $\sigma$ & 0.326 & 0.605 & 0.5 \\
\hline
\end{tabular}

Table 6 Intrinsic parameter comparison for the experiment with the Checkerboard dataset. For each statistic, the top row corresponds to the value for cameras with $f=50 \mathrm{~mm}$ (equivalent in $35 \mathrm{~mm}$ film) and the bottom row corresponds to cameras with $f=100 \mathrm{~mm}$. Absolute data are given in pixels. Relative data are given with respect to a half of the image diagonal.

\begin{tabular}{llll}
\hline Statistic & $\begin{array}{l}\text { Three-Homography } \\
\text { algorithm }\end{array}$ & $\begin{array}{l}\text { Euclidean BA (Node D } \\
\text { in Fig. 4) }\end{array}$ & $\begin{array}{l}\text { Mixed BA (Node D' } \\
\text { in Fig. 4) }\end{array}$ \\
\hline Mean focal length $\alpha$ & 1842 & 1846.8 & 1846.1 \\
& 3565 & 3555 & 3556.7 \\
$\alpha$ standard deviation & 19.82 & 3.95 & 7.67 \\
Mean pp $\left(u_{0}, v_{0}\right)$ & 47.33 & 20.29 & 32.46 \\
& $(625.2,474.3)$ & $(622.8,484.4)$ & $(625.29,486.13)$ \\
Mean dist. pp. to mean pp. & $(647.4,527.3)$ & $(604.1,518.8)$ & $(611.57,520.84)$ \\
& 19.72 & 5.38 & 8.6 \\
Std. dev. of dist. to mean pp. & 59.73 & 21.8 & 27.88 \\
& 12.96 & 3.58 & 6.4 \\
Dist. mean pp. to image center & 36.11 & 8.61 & 16.82 \\
& $15.83(1.98 \%)$ & $17.7(2.21 \%)$ & $15.93(2 \%)$ \\
\hline
\end{tabular}

A total of 283 points were matched across the images, with an average of 234 visible points per image, the checkerboard calibration rig consisting of 189 points. The matched points were taken as input of the algorithms summarized in Fig. 5. Due to the difficulty of using projective factorization with occluded points, this module has been substituted by one iteration of projective bundle adjustment [12, p. 423]. The residual RMS reprojection errors are shown in Table 5 . The small value of this parameter after projective bundle adjustment ( 0.31 pixels) reveals that the points were accurately detected and that for the two chosen focal lengths the effect of radial distortion can be neglected. Furthermore, from the residual reprojection error after projective bundle adjustment the noise in the point positions can be estimated using formulas (67) as $\sigma=0.326$ pixels. So the signal-to-noise ratio is of the order of $2 \times 10^{3}$, i.e., about eight times the minimum considered in the simulations.

Due to the bad performance observed in Sect. 5.2 of the algorithm associated to the node D" (Fig. 4), it was decided to exclude this algorithm from the real data intrinsic parameters comparison. Therefore, the comparison shown in Table 6 only involves nodes D, D' and the parameters estimated through the rig pattern. For the latter we use algorithm in [12, p. 211], which recovers the intrinsic parameters by linear estimation of the IAC from three homographies determined by the projected rig points, without requiring the knowledge of the projection matrix. We have modified this threehomography algorithm, imposing that the computed IACs are consistent with the square pixel hypothesis.

The digital camera used to acquire the images has a CCD sensor of $8.8 \times 6.6 \mathrm{~mm}$, which implies a (square) pixel size of $6.875 \mu \mathrm{m}$ for the image size in this dataset. A $50 \mathrm{~mm}$ focal length in a $35 \mathrm{~mm}$ film sensor corresponds to a focal length of $12.71 \mathrm{~mm}$ in our sensor size, i.e., 1849 pixels given the known pixel size. Such value is very close to those obtained by the different algorithms tested (first row of Table 6).

Table 6 also shows a strong agreement between the values of the intrinsic parameters obtained through the 

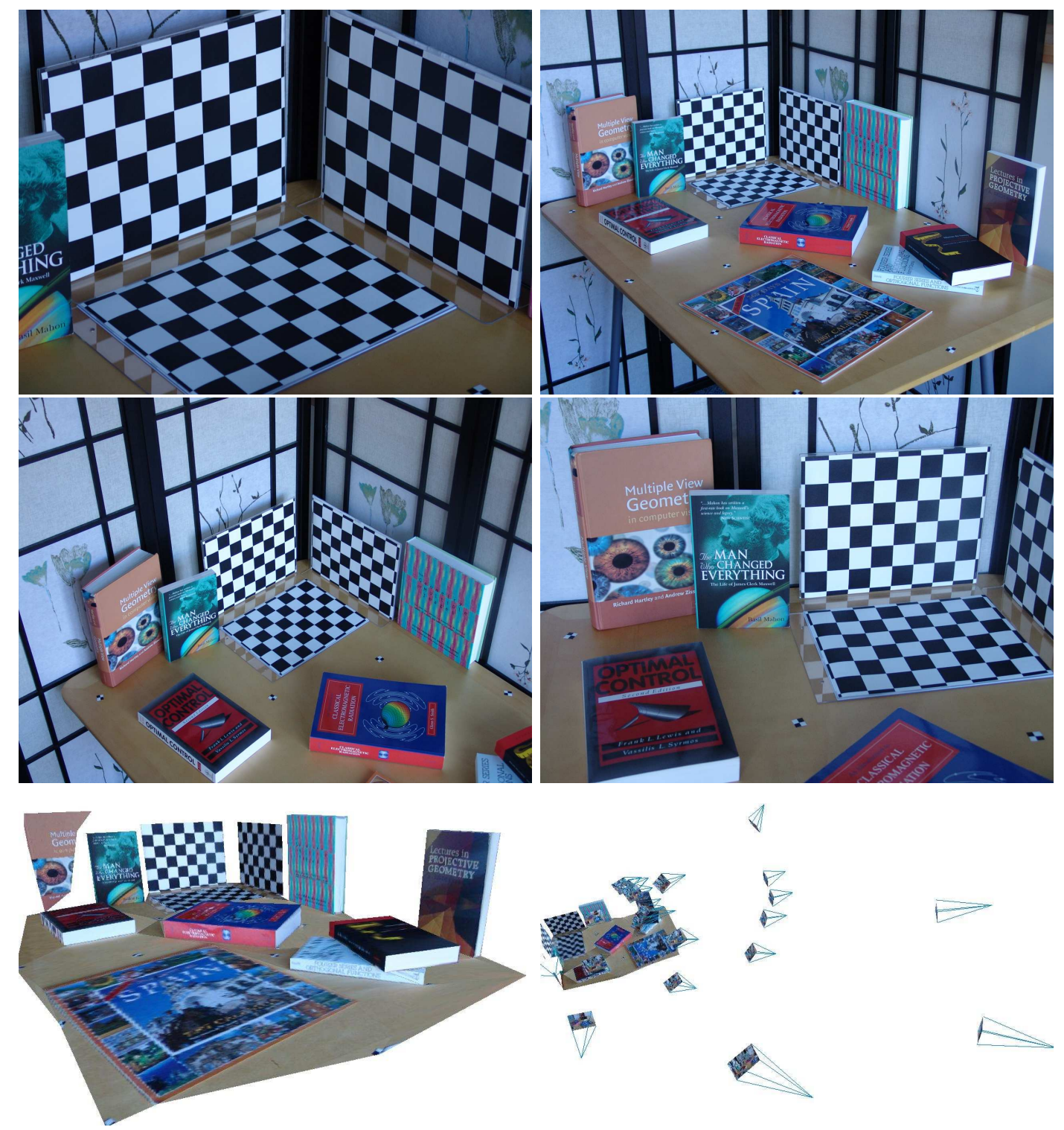

Fig. 7 Four images and two views of the reconstructed Checkerboard scene.

proposed methods and the values derived from the calibration rig. The three-homography algorithm estimates the intrinsic parameters of every camera independently of the rest of the cameras, solely based on the $2 \mathrm{D}$ rig point correspondences. This might explain why the standard deviations of this calibration algorithm are larger than those observed for the two other algorithms in Table 6 .

Figure 7 shows two views of the VRML reconstructed scene corresponding to the algorithm of mixed bundle adjustment.

We also present two more reconstructions, for Books and Kings' Courtyard. These have a smaller signal-tonoise ratio than Checkerboard, as evidences the estimated noise standard deviations in Table 5.
The Books dataset consists of 18 images of $640 \times$ 480 pixels. A partial reconstruction of this scene was obtained by selecting 76 points, with an average of 56 simultaneously visible. In this experiment, zoom was randomly changed for the different images.

The third dataset comprises 23 images of $1024 \times 768$ pixels of the Kings' Courtyard of the Royal Monastery of San Lorenzo de El Escorial (Madrid, Spain), from which 443 point correspondences were selected, with an average of 372 points per image simultaneously visible.

Figures 8 and 9 show respectively two views of these two VRML reconstructed scenes corresponding to the algorithm of minimization of the error in the pixel shape. 


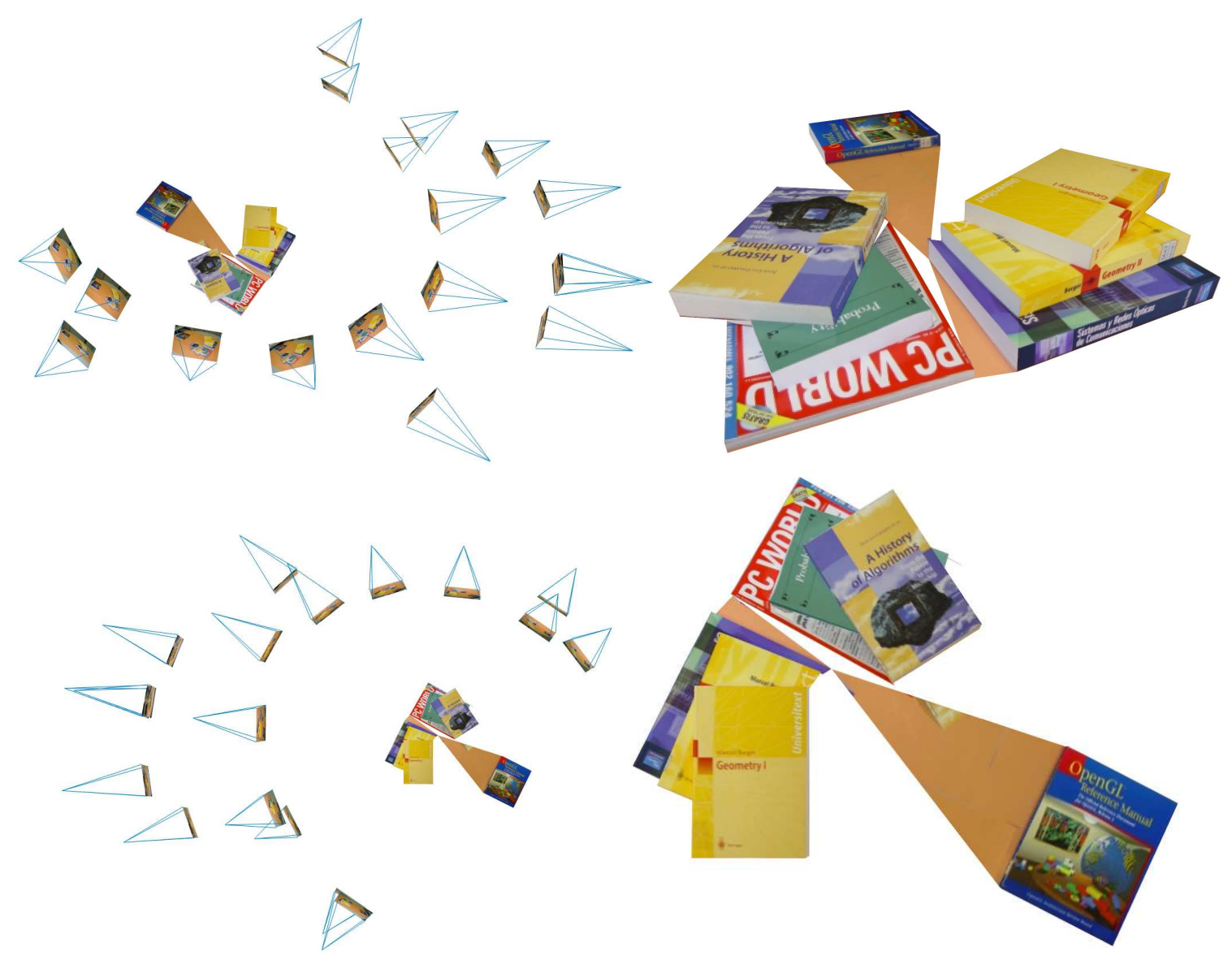

Fig. 8 Four views of the first reconstructed 3D scene. The ones on the left shows camera positions.

\section{Conclusions}

This paper provides an algorithm-oriented reformulation of the set of lines intersecting the absolute conic. This object is a quadric in a five-dimensional projective space called absolute quadratic complex (AQC).

We have provided a self-contained matrix formulation of the AQC. New properties of the AQC have been presented to obtain new autocalibration algorithms. The new results include closed-form expressions for the skewangle of the camera (53), the pixel aspect ratio (54) and the principal point location (55) in terms of the AQC. We propose a new algorithm to obtain the DAQ from the AQC using straightforward matrix operations in Sect. 3.9. We have provided a sound mathematical foundation of the computation of an Euclidean-upgrading homography from the AQC proposed in [20]. We have also characterized (equations (27)) the $6 \times 6$ matrices acting on lines which are induced by a spatial homography, completing a result given in [2]. A mathematical proof of the fact that the operation attaching to each spatial homography its line homography is invariant under transposition (equation (28)) has been given as well, which is an algebraic translation of the self-dual nature of lines in 3D-space.

New autocalibration algorithms, Mixed Bundle Adjustment and Minimization of Error in Pixel Shape, are proposed and compared with some other alternatives. Our main conclusion is that the Minimization of Error in Pixel Shape followed by Euclidean Bundle Adjustment provides the best result, although Mixed Bundle Adjustment produces almost equivalent results with a lower computational cost. The saturation phenomenon on the number of cameras has been shown.

Applicability of the algorithms to obtain 3D reconstructions with real images obtained with cameras with known pixel shape and otherwise arbitrarily varying intrinsic parameters has been empirically tested.

\section{A Appendix: Proofs}

\section{A.1 Properties of antisymmetric matrices}

As antisymmetric matrices are of even rank, a $4 \times 4$ antisymmetric matrix can only have rank zero, two, or four, so that non-null $4 \times 4$ singular antisymmetric ma- 

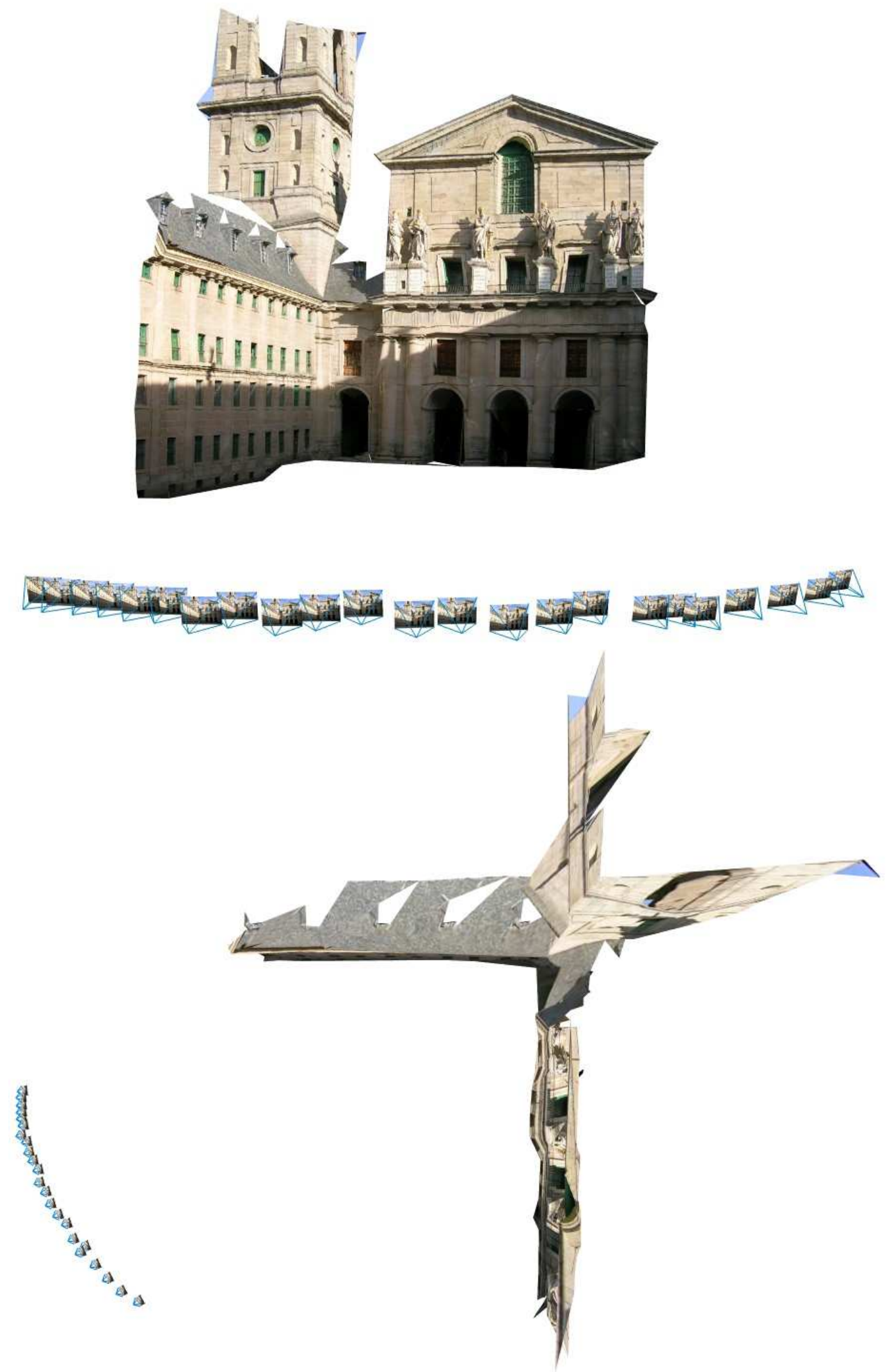

Fig. 9 Two views of the second reconstructed 3D scene (Kings' Courtyard of El Escorial monastery, Madrid) showing camera positions. 
trices can only be of rank two. We state this explicitly for further reference.

Remark A1 Non-null $4 \times 4$ singular antisymmetric matrices are of rank two.

Let A be a singular non-null antisymmetric matrix and let us take two different vectors $\mathbf{u}$ and $\mathbf{v}$ spanning its kernel. Let us consider a change of coordinates $\mathbf{p}^{\prime}=$ Hp, so that $\mathbf{u}^{\prime}=(1,0,0,0)$ and $\mathbf{v}^{\prime}=(0,1,0,0)$. The antisymmetric matrix $\mathrm{A}^{\prime}=\mathrm{H}^{-\top} \mathrm{AH}^{-1}$ satisfies $\mathrm{A}^{\prime} \mathbf{u}^{\prime}=0$, $A^{\prime} \mathbf{v}^{\prime}=0$, which imply that all the entries of $A^{\prime}$ vanish excepting $\mathrm{A}_{3,4}^{\prime}=-\mathrm{A}_{4,3}^{\prime}$. Therefore $\mathrm{A}^{\prime}$ is defined up to a scalar factor and so is $\mathrm{A}$. So we have:

Remark A2 A rank-two $4 \times 4$ antisymmetric matrix is determined by its kernel up to a proportionality constant.

\section{A.2 Incidence between lines in terms of Plücker} matrices

Two lines $l_{2}$ and $l_{3}$, given by points $\mathbf{p}_{1}, \mathbf{q}_{1}$ and $\mathbf{p}_{2}, \mathbf{q}_{2}$ respectively, will intersect if and only if $\operatorname{det}\left(\mathbf{q}_{1}, \mathbf{p}_{2}, \mathbf{q}_{2}, \mathbf{p}_{1}\right)=$ 0 . But this determinant is, from (7),

$$
\begin{aligned}
& \operatorname{det}\left(\mathbf{q}_{1}, \mathbf{p}_{2}, \mathbf{q}_{2}, \mathbf{p}_{1}\right) \\
& =\mathbf{q}_{1}^{\top} \mathrm{M}^{*}\left(\mathbf{p}_{2}, \mathbf{q}_{2}\right) \mathbf{p}_{1} \\
& =\frac{1}{2}\left[\mathbf{q}_{1}^{\top} \mathrm{M}^{*}\left(\mathbf{p}_{2}, \mathbf{q}_{2}\right) \mathbf{p}_{1}-\mathbf{p}_{1}^{\top} \mathrm{M}^{*}\left(\mathbf{p}_{2}, \mathbf{q}_{2}\right) \mathbf{q}_{1}\right] \\
& =\frac{1}{2}\left[\operatorname{trace}\left(\mathbf{q}_{1}^{\top} \mathrm{M}^{*}\left(\mathbf{p}_{2}, \mathbf{q}_{2}\right) \mathbf{p}_{1}\right)-\operatorname{trace}\left(\mathbf{p}_{1}^{\top} \mathrm{M}^{*}\left(\mathbf{p}_{2}, \mathbf{q}_{2}\right) \mathbf{q}_{1}\right)\right] \\
& =\frac{1}{2}\left[\operatorname{trace}\left(\mathbf{p}_{1} \mathbf{q}_{1}^{\top} \mathrm{M}^{*}\left(\mathbf{p}_{2}, \mathbf{q}_{2}\right)\right)-\operatorname{trace}\left(\mathbf{q}_{1} \mathbf{p}_{1}^{\top} \mathrm{M}^{*}\left(\mathbf{p}_{2}, \mathbf{q}_{2}\right)\right)\right] \\
& =\frac{1}{2} \operatorname{trace}\left(\mathbf{p}_{1} \mathbf{q}_{1}^{\top} \mathrm{M}^{*}\left(\mathbf{p}_{2}, \mathbf{q}_{2}\right)-\mathbf{q}_{1} \mathbf{p}_{1}^{\top} \mathrm{M}^{*}\left(\mathbf{p}_{2}, \mathbf{q}_{2}\right)\right) \\
& =\frac{1}{2} \operatorname{trace}\left(\mathrm{M}\left(\mathbf{p}_{1}, \mathbf{q}_{1}\right) \mathrm{M}^{*}\left(\mathbf{p}_{2}, \mathbf{q}_{2}\right)\right) \\
& =\frac{1}{2} \operatorname{trace}\left(\mathrm{L}_{1} \mathrm{~L}_{2}^{*}\right)=0 .
\end{aligned}
$$

\section{A.3 Plücker coordinates and linear mappings}

Now we prove the properties of L-matrices stated in Sect. 2.1. The corresponding properties of $\mathrm{L}^{*}$-matrices result from point-plane projective duality.

The point coordinate change $\mathbf{p}^{\prime}=\mathrm{Hp}$ induces the change of Plücker coordinates

$$
\ell_{\mathrm{M}(\mathrm{H} \mathbf{p}, \mathrm{H} \mathbf{q})}=\ell_{\mathrm{HM}(\mathbf{p}, \mathbf{q}) \mathrm{H}^{\top}}=\tilde{\mathrm{H}} \boldsymbol{\ell}_{\mathrm{M}(\mathbf{p}, \mathbf{q})}
$$

To obtain the $k$-th column of $\tilde{\mathrm{H}}$ we have to compute the new Plücker coordinates of the line with original Plücker coordinates given by the $k$-th element of the canonical basis of $\mathbf{C}^{6}$. Denoting by $\mathbf{h}_{l}$ the columns of $\mathrm{H}$ and using (21) we have that $\boldsymbol{\ell}_{\mathrm{M}\left(\mathrm{He}_{i}, \mathrm{He} \mathbf{e}_{j}\right)}=\boldsymbol{\ell}_{\mathrm{M}\left(\mathbf{h}_{i}, \mathbf{h}_{j}\right)}=$ $\mathbf{h}_{i} \wedge \mathbf{h}_{j}=\tilde{\mathrm{H}} \boldsymbol{\ell}_{\mathrm{M}\left(\mathbf{e}_{i}, \mathbf{e}_{j}\right)}$. From this equation and (14), we obtain the columns of $\tilde{\mathrm{H}}$ :

$$
\begin{aligned}
\tilde{\mathrm{H}}=\left(\mathbf{h}_{3} \wedge \mathbf{h}_{4}\right. & \mathbf{h}_{1} \wedge \mathbf{h}_{4} \quad \mathbf{h}_{2} \wedge \mathbf{h}_{4} \quad \mathbf{h}_{3} \wedge \mathbf{h}_{1} \\
\mathbf{h}_{2} \wedge \mathbf{h}_{3} & \left.\mathbf{h}_{1} \wedge \mathbf{h}_{2}\right) .
\end{aligned}
$$

The matrices of this form have the property $\tilde{\mathrm{H}}^{\top} \Omega \tilde{\mathrm{H}}=$ $\rho \Omega$. This is geometrically clear, since $\tilde{\mathrm{H}}$ maps Plücker coordinates onto Plücker coordinates so it must preserve $\Omega$. However, a direct proof will also allow us to compute the scaling factor $\rho$. We observe from (70) that the entries of the matrix $\tilde{\mathrm{H}}^{\top} \Omega \tilde{\mathrm{H}}$ are of the form

$$
\left(\mathbf{h}_{i} \wedge \mathbf{h}_{j}\right)^{\top} \Omega\left(\mathbf{h}_{k} \wedge \mathbf{h}_{l}\right)
$$

Then, making use of the relationship

$(\mathbf{x} \wedge \mathbf{y})^{\top} \Omega(\mathbf{z} \wedge \mathbf{w})=\operatorname{det}(\mathbf{x}, \mathbf{y}, \mathbf{z}, \mathbf{w})$

that stems from (20) and (68), we can compute

$\tilde{\mathrm{H}}^{\top} \Omega \tilde{\mathrm{H}}=\operatorname{det}(\mathrm{H}) \Omega$.

Note that the construction of $\tilde{\mathrm{H}}$ from $\mathrm{H}$ can be done regardless of the regularity of $\mathrm{H}$ and that a continuity argument shows that formula (72) holds true also for singular matrices.

A.4 A necessary and sufficient condition for a $6 \times 6$ matrix A to be of the form $\tilde{\mathrm{H}}$

A $6 \times 6$ matrix $A=\left(\mathbf{a}_{1}, \ldots, \mathbf{a}_{6}\right)$ is of the form $A=\tilde{H}$ for some regular matrix $\mathrm{H}$ if and only if

$\mathrm{A}^{\top} \Omega \mathrm{A} \sim \Omega$
$\mathrm{L}_{1}^{*} \mathrm{~L}_{2} \mathrm{~L}_{3}^{*}=0$

where the $\mathrm{L}_{i}$ are Plücker matrices defined by the condition $\ell_{\mathrm{L}_{i}}=\mathbf{a}_{i}$, whose existence is warranted by (73) as explained below.

To prove this result we observe, using (73), that the columns $\mathbf{a}_{1}, \ldots, \mathbf{a}_{6}$ of $\mathbf{A}$ are Plücker coordinates of lines, since $\mathbf{a}_{i}^{\top} \Omega \mathbf{a}_{i}=0$, and each line intersects all the others but one, as $\mathbf{a}_{i}^{\top} \Omega \mathbf{a}_{j}=0$ for $j \neq 7-i$. Since $\mathbf{a}_{1}, \mathbf{a}_{2}$ and $\mathbf{a}_{3}$ intersect pairwise, they are either coplanar or incident in a common point. If $\mathrm{A}=\tilde{\mathrm{H}}$ for some $\mathrm{H}$, we are in the second case according to (25). Equation (74) (see Table 1) characterizes this configuration. Now, a straightforward combinatorial argument shows that the last three columns of A represent coplanar lines that form together with the first three lines the edges of a tetrahedron. Let us denote by $\mathbf{p}_{1}, \ldots, \mathbf{p}_{4}$ a set of vectors representing the vertices of this tetrahedron, so that 
$\mathbf{p}_{1}=\mathbf{a}_{2} \cap \mathbf{a}_{4} \cap \mathbf{a}_{6}, \mathbf{p}_{2}=\mathbf{a}_{3} \cap \mathbf{a}_{5} \cap \mathbf{a}_{6}, \mathbf{p}_{3}=\mathbf{a}_{1} \cap \mathbf{a}_{4} \cap \mathbf{a}_{5}$, $\mathbf{p}_{4}=\mathbf{a}_{1} \cap \mathbf{a}_{2} \cap \mathbf{a}_{3}$. Therefore we have

$$
\begin{aligned}
& \mathbf{A}=\left(\lambda_{1} \mathbf{p}_{3} \wedge \mathbf{p}_{4} \quad \lambda_{2} \mathbf{p}_{1} \wedge \mathbf{p}_{4} \quad \lambda_{3} \mathbf{p}_{2} \wedge \mathbf{p}_{4} \quad \lambda_{4} \mathbf{p}_{3} \wedge \mathbf{p}_{1}\right. \\
& \left.\lambda_{5} \mathbf{p}_{2} \wedge \mathbf{p}_{3} \quad \lambda_{6} \mathbf{p}_{1} \wedge \mathbf{p}_{2}\right)
\end{aligned}
$$

for some coefficients $\lambda_{i}$. Defining

$\mathrm{H}=\left(\frac{\sqrt{\lambda_{4}} \sqrt{\lambda_{6}}}{\sqrt{\lambda_{5}}} \mathbf{p}_{1} \frac{\sqrt{\lambda_{5}} \sqrt{\lambda_{6}}}{\sqrt{\lambda_{4}}} \mathbf{p}_{2} \frac{\sqrt{\lambda_{4}} \sqrt{\lambda_{5}}}{\sqrt{\lambda_{6}}} \mathbf{p}_{3} \lambda_{1} \frac{\sqrt{\lambda_{6}}}{\sqrt{\lambda_{4}} \sqrt{\lambda_{5}}} \mathbf{p}_{4}\right)$

it follows that $\mathrm{A}=\tilde{\mathrm{H}}$. To check this it is necessary to use the identities $\lambda_{1} \lambda_{6}=\lambda_{2} \lambda_{5}=\lambda_{3} \lambda_{4}$, which follow from $\mathbf{a}_{1}^{\top} \Omega \mathbf{a}_{6}=\mathbf{a}_{2}^{\top} \Omega \mathbf{a}_{5}=\mathbf{a}_{3}^{\top} \Omega \mathbf{a}_{4}$ and (71). This completes the proof.

We can obtain an interesting alternative formula for $\tilde{\mathrm{H}}$ using $\mathrm{M}^{*}$ matrices. We recall that, given a cooordinate change $\mathbf{p}^{\prime}=\mathrm{H} \mathbf{p}$, the corresponding coordinate change for planes is $\boldsymbol{\alpha}^{\prime}=\mathrm{H}^{-\top} \boldsymbol{\alpha}$, and the resulting coordinate change for Plücker coordinates will be

$$
\begin{aligned}
\boldsymbol{\ell}_{\mathrm{M}^{*}\left(\boldsymbol{\alpha}^{\prime}, \boldsymbol{\beta}^{\prime}\right)} & =\boldsymbol{\ell}_{\mathrm{M}^{*}\left(\mathrm{H}^{-\top} \boldsymbol{\alpha}, \mathrm{H}^{-\top} \boldsymbol{\beta}\right)} \\
& \stackrel{(13)}{=} \boldsymbol{\ell}_{\operatorname{det}\left(\mathrm{H}^{-1}\right) \mathrm{H}^{*}(\boldsymbol{\alpha}, \boldsymbol{\beta}) \mathrm{H}^{\top}} \\
& =\operatorname{det}\left(\mathrm{H}^{-1}\right) \boldsymbol{\ell}_{\mathrm{H} \mathrm{M}^{*}(\boldsymbol{\alpha}, \boldsymbol{\beta}) \mathrm{H}^{\top}} \\
& \stackrel{(69)}{=} \operatorname{det}\left(\mathrm{H}^{-1}\right) \tilde{\mathrm{H}} \boldsymbol{\ell}_{\mathrm{M}^{*}(\boldsymbol{\alpha}, \boldsymbol{\beta}) .}
\end{aligned}
$$

Let us define

$\hat{\mathrm{H}}=\operatorname{det}\left(\mathrm{H}^{-1}\right) \tilde{\mathrm{H}}$.

From (75), $\hat{\mathrm{H}}^{-1} \boldsymbol{\ell}_{\mathrm{M}^{*}\left(\boldsymbol{\alpha}^{\prime}, \boldsymbol{\beta}^{\prime}\right)}=\boldsymbol{\ell}_{\mathrm{M}^{*}\left(\mathrm{H}^{\top} \boldsymbol{\alpha}^{\prime}, \mathrm{H}^{\top} \boldsymbol{\beta}^{\prime}\right)}$. Therefore, denoting by $\mathbf{v}_{i}^{\top}$ the rows of $\mathrm{H}$, we can prove in a similar way to (70) that

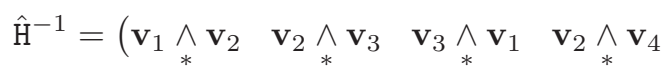

$$
\begin{aligned}
& \left.\mathbf{v}_{1} \bigwedge_{*}^{\wedge} \mathbf{v}_{4} \quad \mathbf{v}_{3} \stackrel{*}{*}_{\mathbf{v}_{4}}\right) .
\end{aligned}
$$

Using (72) we obtain $\tilde{\mathrm{H}}^{\top}=\operatorname{det}(\mathrm{H}) \Omega \tilde{\mathrm{H}}^{-1} \Omega$, and from (76) and (22),

$$
\begin{aligned}
& \tilde{\mathrm{H}}^{\top}=\Omega \hat{\mathrm{H}}^{-1} \Omega=\left(\mathbf{v}_{3} \wedge \mathbf{v}_{4} \quad \mathbf{v}_{1} \wedge \mathbf{v}_{4} \quad \mathbf{v}_{2} \wedge \mathbf{v}_{4} \quad \mathbf{v}_{3} \wedge \mathbf{v}_{1}\right. \\
& \left.\mathbf{v}_{2} \wedge \mathbf{v}_{3} \quad \mathbf{v}_{1} \wedge \mathbf{v}_{2}\right),
\end{aligned}
$$

where we have used that right-multiplying a matrix by $\Omega$ reverts the order of the columns. Comparison of (78) with (70) yields $\tilde{\mathrm{H}}^{\top}=\widetilde{\mathrm{H}^{\top}}$ (cf. [2]).

\section{A.5 Plücker coordinates and projections}

Let us consider a camera given by a projection matrix $\mathrm{P}=\left(\boldsymbol{\pi}_{1}, \boldsymbol{\pi}_{2}, \boldsymbol{\pi}_{3}\right)^{\top}$. A point $\mathbf{X} \in \mathbb{P}^{3}$ belongs to the backprojected line of $\mathbf{x}=(u, v, w)^{\top}$ if and only if $\mathbf{x} \sim \mathbf{P X}$, or equivalently, $\mathbf{x} \times \mathbf{P X}=\mathbf{0}$, i.e.,

$$
\begin{aligned}
\mathbf{X} \times \mathbf{P} \mathbf{X} & =[\mathbf{x}]_{\times} \mathbf{P} \mathbf{X}=\left(\begin{array}{ccc}
0 & -w & v \\
w & 0 & -u \\
-v & u & 0
\end{array}\right)\left(\begin{array}{l}
\boldsymbol{\pi}_{1}^{\top} \\
\boldsymbol{\pi}_{2}^{\top} \\
\boldsymbol{\pi}_{3}^{\top}
\end{array}\right) \mathbf{X} \\
& =\left(\begin{array}{l}
v \boldsymbol{\pi}_{3}^{\top}-w \boldsymbol{\pi}_{2}^{\top} \\
w \boldsymbol{\pi}_{1}^{\top}-u \boldsymbol{\pi}_{3}^{\top} \\
u \boldsymbol{\pi}_{2}^{\top}-v \boldsymbol{\pi}_{1}^{\top}
\end{array}\right) \mathbf{X}=\mathbf{0} .
\end{aligned}
$$

Therefore, the planes $\boldsymbol{\alpha}_{1}=v \boldsymbol{\pi}_{3}-w \boldsymbol{\pi}_{2}, \boldsymbol{\alpha}_{2}=w \boldsymbol{\pi}_{1}-u \boldsymbol{\pi}_{3}$ and $\boldsymbol{\alpha}_{3}=u \boldsymbol{\pi}_{2}-v \boldsymbol{\pi}_{1}$ define the pencil given by the backprojected line of $\mathbf{x}$. The Plücker coordinates of this line will be any of the following, as long as it is not null:

$$
\begin{aligned}
& \boldsymbol{\alpha}_{2} \wedge \boldsymbol{\alpha}_{3}=\left[u\left(\boldsymbol{\pi}_{2} \wedge \boldsymbol{\pi}_{3}\right)+v\left(\boldsymbol{\pi}_{3} \wedge \boldsymbol{\pi}_{1}\right)+w\left(\boldsymbol{\pi}_{1} \wedge \boldsymbol{\pi}_{2}\right)\right] u \\
& \boldsymbol{\alpha}_{3} \wedge_{*}^{*} \boldsymbol{\alpha}_{1}=\left[u\left(\boldsymbol{\pi}_{2} \wedge_{*}^{*} \boldsymbol{\pi}_{3}\right)+v\left(\boldsymbol{\pi}_{3} \wedge_{*}^{*} \boldsymbol{\pi}_{1}\right)+w\left(\boldsymbol{\pi}_{1} \wedge_{*}^{*} \boldsymbol{\pi}_{2}\right)\right] v \\
& \boldsymbol{\alpha}_{1} \wedge_{*}^{\wedge} \boldsymbol{\alpha}_{2}=\left[u\left(\boldsymbol{\pi}_{2} \wedge_{*}^{*} \boldsymbol{\pi}_{3}\right)+v\left(\boldsymbol{\pi}_{3} \wedge_{*}^{*} \boldsymbol{\pi}_{1}\right)+w\left(\boldsymbol{\pi}_{1} \wedge_{*}^{\wedge} \boldsymbol{\pi}_{2}\right)\right] w
\end{aligned}
$$

At least one of the $\wedge$ products above must be nonzero, for if the three $\boldsymbol{\alpha}_{i}^{*} \wedge \boldsymbol{\alpha}_{j}$ vanish, we will have $\boldsymbol{\alpha}_{1} \sim$ $\boldsymbol{\alpha}_{2} \sim \boldsymbol{\alpha}_{3}$ and the back-projected line would not be welldefined. Hence the common factor $u\left(\boldsymbol{\pi}_{2} \wedge \boldsymbol{\pi}_{3}\right)+v\left(\boldsymbol{\pi}_{3} \wedge\right.$ $\left.\boldsymbol{\pi}_{1}\right)+w\left(\boldsymbol{\pi}_{1} \wedge \boldsymbol{\pi}_{2}\right)$ must be nonzero and correspond to the Plücker coordinates of the back-projected line. Thus the mapping from image points to back-projected lines is given by equation $\ell=\mathcal{P}^{\top} \mathbf{x}$, where $\mathcal{P}^{\top}=\left(\boldsymbol{\pi}_{2} \wedge_{*}\right.$ $\left.\boldsymbol{\pi}_{3} \boldsymbol{\pi}_{3} \wedge \boldsymbol{\pi}_{1} \boldsymbol{\pi}_{1} \wedge \boldsymbol{\pi}_{2}\right)$.

Given the space line $\boldsymbol{\ell}$, a point $\mathbf{x}$ of the image plane will belong to the projection of $\ell$ if and only if its back-projected line $\mathcal{P}^{\top} \mathbf{x}$ intersects $\boldsymbol{\ell}$, i.e., $\left(\mathcal{P}^{\top} \mathbf{x}\right)^{\top} \Omega \boldsymbol{\ell}=$ $\mathbf{x}^{\top} \mathcal{P} \Omega \boldsymbol{\ell}=0$. Therefore, the projection of $\boldsymbol{\ell}$ has coordinates $\mathcal{P} \Omega \boldsymbol{\ell}$, so that the matrix of the mapping from lines in space to their projections is $\mathcal{P} \Omega$ (cf. [9, p. 183]).

\section{A.6 Factorization of $\Sigma$}

Let

$\Sigma=\mathrm{G}^{\top} \Sigma_{\text {euc }} \mathrm{G}=\mathrm{G}^{\prime \top} \Sigma_{\text {euc }} \mathrm{G}^{\prime}$

be two decompositions of $\Sigma$. Then $\mathrm{V}^{\top} \Sigma_{\text {euc }} \mathrm{V}=\Sigma_{\text {euc }}$ where $\mathrm{V}=\mathrm{GG}^{\prime-1}$. Writing $\mathrm{V}=\left(\begin{array}{ll}\mathrm{A} & \mathrm{B} \\ \mathrm{C} & \mathrm{D}\end{array}\right)$, it is easy to check that $\mathrm{A}$ must be orthogonal and $\mathrm{B}=0$.

\section{A.7 Proof of Theorem 1}

We first check that $\mathbf{r}_{i}$ are the Plücker coordinates of three concurrent lines. If we define the matrix $\mathcal{R}=$ $\left(\mathbf{r}_{1}, \mathbf{r}_{2}, \mathbf{r}_{3}\right)$, we have $\Sigma=\mathrm{G}^{\top} \Sigma_{\text {euc }} \mathrm{G}=\mathcal{R} \mathcal{R}^{\top}$. Therefore $\mathcal{R}$ must be a rank-three matrix, since so is $\Sigma$. 
From (39), we have $\Sigma \Omega \Sigma=\mathrm{G}^{\top} \Sigma_{\text {euc }} \mathrm{G} \Omega \mathrm{G}^{\top} \Sigma_{\text {euc }} \mathrm{G}=0$, which, due to the regularity of $G$ and the fact that $\Sigma_{\text {euc }} \mathrm{G}=\left(\mathcal{R}, 0_{6 \times 3}\right)^{\top}$, implies $\mathcal{R}^{\top} \Omega \mathcal{R}=0$, so that for $i=1,2,3$ we have $\mathbf{r}_{i}^{\top} \Omega \mathbf{r}_{j}=0$. These relationships mean, according to (19) and (20), that the $\mathbf{r}_{i}$ represent Plücker coordinates of lines intersecting pairwise.

Therefore there are two possible geometrical configurations for the lines represented by the $\mathbf{r}_{i}$ : either they are non-coplanar lines intersecting in a common point or they are three lines in a common plane pairwise intersecting in three different points. Being $\mathcal{R}$ rank-three, these two possibilities are mutually excluding. To determine the actual configuration, we will make use of the fact that the kernel of $\Sigma$ are the lines of a plane (the plane at infinity, see the comment after formula (39)). Let us first observe that the kernel of $\Sigma \Omega$ consists exactly of those lines intersecting the three lines $\mathbf{r}_{i}$. To check this, take $\mathbf{s}$ to represent any line intersecting the $\mathbf{r}_{i}$, so that $\mathbf{r}_{i}^{\top} \Omega \mathbf{s}=\mathbf{0}, i=1,2,3$. Therefore $\mathcal{R}^{\top} \Omega \mathbf{s}=\mathbf{0}$, and then $\mathcal{R \mathcal { R }}^{\top} \Omega \mathbf{s}=\Sigma \Omega \mathbf{s}=\mathbf{0}$, so $\mathbf{s} \in \operatorname{ker}(\Sigma \Omega)$. Since both $\operatorname{ker}(\Sigma \Omega)$ and the set of the lines that intersect the $\mathbf{r}_{i}$ are linear spaces of the same dimension (being the latter either the set of lines through the common point or in the common plane), they coincide.

As ker $\Sigma$ are the lines of a plane, $\operatorname{ker}(\Sigma \Omega)=\Omega \operatorname{ker} \Sigma$ is a star of lines through a point (22). We conclude that the $\mathbf{r}_{i}$ share a common point $\mathbf{v}_{4}$. Let us take three vectors $\mathbf{v}_{i}, i=1,2,3$, such that $\mathbf{r}_{1}=\mathbf{v}_{3} \wedge \mathbf{v}_{4}, \mathbf{r}_{2}=$ $\mathbf{v}_{1} \wedge \mathbf{v}_{4}, \mathbf{r}_{3}=\mathbf{v}_{2} \wedge \mathbf{v}_{4}$. We define the matrix $\mathrm{H}^{\top}=$ $\left(\mathbf{v}_{1}, \mathbf{v}_{2}, \mathbf{v}_{3}, \mathbf{v}_{4}\right)$ so we can write our factorization as

$$
\begin{aligned}
& \Sigma= \mathcal{R} \mathcal{R}^{\top}=\left(\mathbf{v}_{3} \wedge \mathbf{v}_{4} \mathbf{v}_{1} \wedge \mathbf{v}_{4} \mathbf{v}_{2} \wedge \mathbf{v}_{4}\right) \\
& \cdot\left(\mathbf{v}_{3} \wedge \mathbf{v}_{4} \mathbf{v}_{1} \wedge \mathbf{v}_{4} \mathbf{v}_{2} \wedge \mathbf{v}_{4}\right)^{\top} \\
&=\widetilde{\mathrm{H}}^{\top} \Sigma_{\text {euc }} \widetilde{\mathrm{H}},
\end{aligned}
$$

where formulas (28) and (25) have been used. Therefore $\mathrm{H}$ is the matrix of the change of basis to a Euclidean coordinate system, i.e., points satisfy $\mathbf{X}_{\text {euc }}=H \mathbf{X}$.

\section{A.8 Proof of Theorem 2}

The necessity of the conditions follows from Sect. 3.4, equation (39), and the subsequent discussion.

Sufficiency results from the fact that these conditions are exactly those used in the proof of Theorem 1 to obtain a change of coordinates that converts $\Sigma$ into $\Sigma_{\text {euc }}$.

Acknowledgements This work has been partly supported by the Spanish Administration agencies CDTI and CICYT under projects CENIT-VISION 2007-1007 and TEC2007-67764 respectively.

\section{References}

1. L. Agapito, E. Hayman, and I. Reid. Self-calibration of rotating and zooming cameras. International Journal of Computer Vision, 45:107-127, 2001.

2. A. Bartoli and P. Sturm. The 3d line motion matrix and alignment of line reconstructions. International Journal of Computer Vision, 57:159-178, 2004.

3. E. Bayro-Corrochano and V. Banarer. A geometric approach for the theory and applications of $3 \mathrm{~d}$ projective invariants. Journal of Mathematical Imaging and Vision, 16(2):131-154, March 2002.

4. M. Berger. Geometry. Springer Verlag, Germany, 1987.

5. S. Bougnoux. From projective to euclidean space under any practical situation, a criticism of self-calibration. In Proc. International Conference on Computer Vision, pages 790-796, Brisbane, Australia, 1998.

6. S. Carlsson. The double algebra: An effective tool for computing invariants in computer vision. In Proc. of the Second Joint European-US Workshop on Applications of Invariance in Computer Vision, pages 145-164, London, UK, 1994. Springer-Verlag.

7. O. Faugeras. What can be seen in three dimensions with an uncalibrated stereo rig. Proc. European Conference on Computer Vision, pages 563-578, 1992.

8. O. Faugeras. Three Dimensional Computer Vision. MIT Press, 1993.

9. O. Faugeras, Q.-T. Luong, and T. Papadopoulou. The Geometry of Multiple Images: The Laws That Govern The Formation of Images of A Scene and Some of Their Applications. MIT Press, Cambridge, MA, USA, 2001.

10. D. A. Forsyth and J. Ponce. Computer Vision: A Modern Approach. Prentice Hall, August 2002.

11. R. Hartley and A. Zisserman. Multiple View Geometry in Computer Vision. Cambridge University Press, second edition, 2003.

12. R. I. Hartley. Estimation of relative camera positions for uncalibrated cameras. In Proc. European Conference on Computer Vision, pages 579-587, London, UK, 1992. Springer-Verlag.

13. A. Heyden. Geometry and algebra of multiple projective transformations. PhD thesis, Lund University, 1995.

14. A. Heyden and K. Åström. Euclidean reconstruction from image sequences with varying and unknown focal length and principal point. In Proc. IEEE Conference on Computer Vision and Pattern Recognition, New York, USA, 1997.

15. F. Kahl, B. Triggs, and K. Åström. Critical motions for auto-calibration when some intrinsic parameters can vary. Journal of Mathematical Imaging and Vision, 13(2):131-146, 2000.

16. Y. Ma, S. Soatto, J. Kosecka, and S. Sastry. An Invitation to 3-D Vision. Springer, 2005.

17. S. J. Maybank and O. D. Faugeras. A theory of selfcalibration of a moving camera. International Journal of Computer Vision, 8(2):123-151, August 1992.

18. M. Pollefeys and L. V. Gool. A stratified approach to metric self-calibration. In Proc. of the IEEE Conference on Computer Vision and Pattern Recognition, pages 407412, June 1997.

19. M. Pollefeys, R. Koch, and L. van Gool. Self-calibration and metric reconstruction in spite of varying and unknown internal camera parameters. International Journal of Computer Vision, 1(32):7-25, 1999.

20. J. Ponce. On computing metric upgrades of projective reconstructions under the rectangular pixel assumption. In 
Second European Workshop on 3D Structure from Multiple Images of Large-Scale Environments, pages 52-67, London, UK, 2001. Springer-Verlag.

21. J. Ponce, K. McHenry, T. Papadopoulo, M. Teillaud, and B. Triggs. On the absolute quadratic complex and its application to autocalibration. In Proc. IEEE Conference on Computer Vision and Pattern Recognition, volume 1, pages 780-787, Washington, DC, USA, 2005.

22. H. Pottman and J. Wallner. Computational Line Geometry. Springer-Verlag New York, Inc., Secaucus, NJ, USA, 2001.

23. W. H. Press, S. A. Teukolsky, W. T. Vetterling, and B. P. Flannery. Numerical Recipes in C: The Art of Scientific Computing. Cambridge University Press, Cambridge, UK, 2nd edition edition, 1993.

24. J. I. Ronda, G. Gallego, and A. Valdés. Camera autocalibration using Plücker coordinates. In International Conference on Image Processing, volume 3, pages 800-803, Genoa, Italy, 2005.

25. J. G. Semple and G. T. Kneebone. Algebraic Projective Geometry. Oxford Classic Texts in the Physical Sciences, Clarendon Press, Oxford, UK, 1952.

26. Y. Seo and A. Heyden. Auto-calibration from the orthogonality constraints. In Proc. International Conference on Pattern Recognition, volume 01, pages 1067-1071, Los Alamitos, CA, USA, 2000.

27. Y. Seo, A. Heyden, and R. Cipolla. A linear iterative method for auto-calibration using the dac equation. In Proc. IEEE Conference on Computer Vision and Pattern Recognition, pages 880-885, Barcelona, Spain, 2001.

28. L. N. Trefethen and D. Bau. Numerical Linear Algebra. SIAM, June 1997.

29. B. Triggs. Autocalibration and the absolute quadric. In Proc. of the IEEE Conference on Computer Vision and Pattern Recognition, pages 609-614, Puerto Rico, USA, June 1997.

30. A. Valdés and J. Ronda. Camera autocalibration and the calibration pencil. Journal of Mathematical Imaging and Vision, 23(2):167-174, 2005.

31. A. Valdés, J. I. Ronda, and G. Gallego. Linear camera autocalibration with varying parameters. In Proc. International Conference on Image Processing, volume 5, pages 3395-3398, Singapore, 2004.

32. A. Valdés, J. I. Ronda, and G. Gallego. The absolute line quadric and camera autocalibration. International Journal of Computer Vision, 66(3):283-303, 2006. 